TOKYO J. MATH.

VOL. 28, No. 2, 2005

\title{
Systematic Singular Triangulations of All Orientable Seifert Manifolds
}

\author{
Taiji TANIGUCHI*, Keiko TSUBOI and Masakatsu YAMASHITA
}

Keio University, Dokkyo Saitama High School and Toyo University

(Communicated by Y. Maeda)

\section{Introduction}

In this paper, we construct singular triangulations [1] of all orientable Seifert manifolds [2]. Especially, we consider singular triangulations with only one vertex, called one-vertex triangulation. Our construction is useful to calculate the state sum type invariant, for example, Turaev-Viro invariant, Turaev-Viro-Ocneanu invariant or Dijkgraaf-Witten invariant; this subject will be seen in forthcoming paper [3]. Also our work is made use of the introduction of a new complexity invariant of closed 3-manifold, see [4].

Let $\mathcal{M}$ be a Seifert manifold and $P$ be a special spine [5] of $\mathcal{M}$. Considering a dual complex for $\mathcal{M}$ relative to $P$, we obtain a one-vertex triangulation of $\mathcal{M}$. Now, how to construct a special spine $P$ of $\mathcal{M}$ ? Our construction is based on the fact that any orientable Seifert manifold is obtained by gluing $M_{n}, J$ and $V_{p, q}$, which are homeomorphic to $\left(S^{2}-\right.$ $\left.\coprod_{i=1}^{n} D_{i}^{2}\right) \times S^{1},\left(S^{1} \times S^{1}-D^{2}\right) \times S^{1}$ and $(p, q)$-type fibered solid torus respectively.

The first step is to make special spines $P_{M_{n}}, P_{J}, P_{V_{p, q}}$ of three compact manifolds $M_{n}$, $J$ and $V_{p, q}$ satisfying the following conditions: each connected component of $\partial M_{n} \cap P_{M_{n}}$, $\partial J \cap P_{J}$ and $\partial V_{p, q} \cap P_{V_{p, q}}$ is the theta-curve shown in Figure 1 and the loop $\gamma \bar{\alpha}$ is a fiber, where $\bar{\alpha}$ means the reverse direction of the edge labeled $\alpha$. As an example, the solid torus $V_{1,1}$

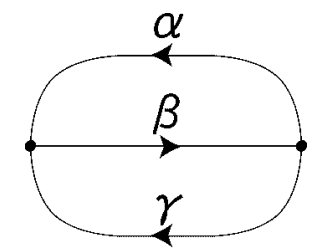

FIGURE 1. A theta-curve $\theta$.

Received May 11, 2004; revised November 10, 2004; revised February 4, 2005

${ }^{*}$ This work is supported in part by the Ministry of Education, Culture, Sport, Science and Technology in Japan under a grant in Aid of the 21st Century Center of Excellence for Integrative Mathematical Sciences: Progress in Mathematics Motivated by Social and Natural Sciences. 
and a special spine $P_{V_{1,1}}$ is shown in Figure 5. The second step is to define fiber preserving homeomorphisms $\varphi_{i}:\left\{\partial J, \partial V_{p, q}\right\} \rightarrow \partial M_{n}$, see $\S 5.4$. Then, we construct any orientable Seifert manifold $\mathcal{M}$ by gluing $J, V_{p, q}$ and $M_{n}$. Furthermore, we show that the polyhedron $P_{\mathcal{M}}$ obtained by gluing $P_{J}, P_{V_{p, q}}$ and $P_{M_{n}}$ by $\left\{\varphi_{i}\right\}$ is a special spine of $\mathcal{M}$. Thus, the dual complex for $\mathcal{M}$ concerning $P_{\mathcal{M}}$ is a one-vertex triangulation of $\mathcal{M}$.

\section{Word diagram of a solid torus}

Let $w=X_{1} X_{2} \cdots X_{n}$ be a sequence of elements $X_{i} \in\{L, R, \bar{L}, \bar{R}\}, i=1,2, \cdots, n$. We call such a sequence $w=w(L, R)$ as a word on the letters $\{L, R\}$. In this section, for a word $w(L, R)$, we will define a word diagram, denoted by $w(L, R)$-diagram. Then, we show that $w(L, R)$-diagram induces an identification map $f_{w(L, R)}$ on $\partial B^{3}$ such that $B^{3} / f_{w(L, R)}$ is homeomorphic to a solid torus.

In $\S 2.1$, we consider the case when the word $w(L, R)$ is the empty word. We use the notation $\phi$ for the empty word. The $\phi$-diagram plays a role of a pit of $w(L, R)$-diagram of a solid torus. In $\S 2.2$, we consider the case when the word $w(L, R)$ is not the empty word.

2.1. The case $w(L, R)=\phi$. Let $D_{\phi}$ be the 2-disc with labels $P, A, Q, \alpha, \beta, \gamma, u$, $v, w$ shown in Figure 2. We call $D_{\phi}$ the $\phi$-disc. It may become clear for the role of labels in $D_{\phi}$, see Remark 2.1.

Suppose the $\phi$ - disc is embedded in $S^{2}$, see Figure 3. Then, the 2-sphere $S^{2}$ is separated into two discs by the circle $\alpha \beta \gamma \bar{\alpha} \bar{\beta} \bar{\gamma}\left(=\partial D_{\phi}\right.$ ), where $\bar{X}$ means the reverse direction of the edge labeled $X$. We denote by $E_{\phi}$ the closed $\operatorname{dics} S^{2} \backslash \operatorname{Int}\left(D_{\phi}\right)$.

The $\phi$-disc gives a natural pasting information $f_{\phi}$ on $S^{2}\left(=\partial B^{3}\right)$ to obtain a compact 3-manifold $B^{3} / f_{\phi}$. The $\phi$-disc consists of $Z_{0}, Z_{1}$ and $Z_{2}$, where $Z_{0}, Z_{1}$ and $Z_{2}$ are sets of 0 -cells, oriented 1-cells and oriented 2-cells respectively. Now, we will explain $Z_{0}, Z_{1}$ and

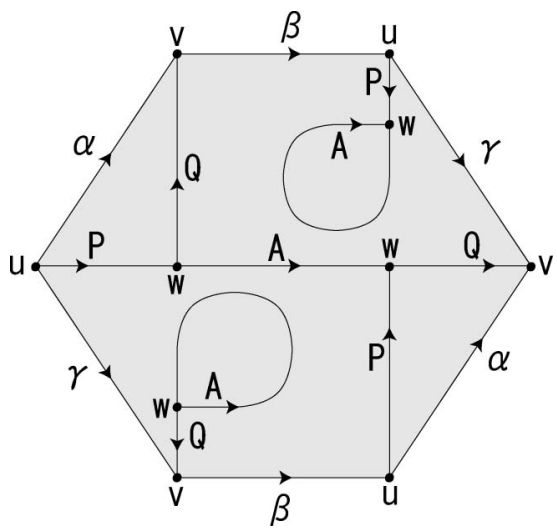

FIGURE 2. $\phi$-disc $D_{\phi}$.

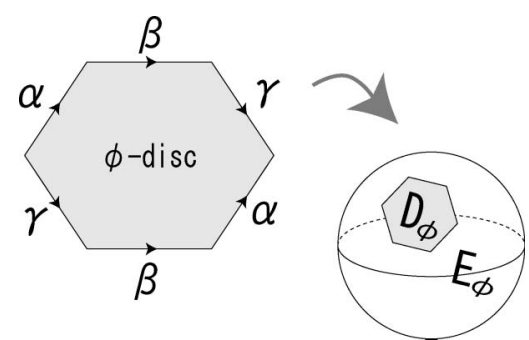

FIGURE 3. $E_{\phi}$. 
$Z_{2}$ and define a pasting information $f_{\phi}$ on $S^{2}$. Although it is only an example, but it must give a suggestion of a general method to obtain a pasting information.

In the $\phi$-disc, there are some 0 -cells with the labels $u, v, w$. We call a 0 -cell a vertex. Thus, the set $Z_{0}$ consists of ten vertices $\left\{u^{(i)}, v^{(i)}, w^{(j)} \mid i=1,2,3\right.$ and $\left.j=1,2,3,4\right\}$, where $X^{(i)}$ is the vertex with the label $X$. Then, the map $\left.f_{\phi}\right|_{Z_{0}}$ is defined by satisfying the following conditions:

1. For any $X \in\{u, v\}$ and $i \in\{1,2,3\}, f_{\phi}\left(X^{(i)}\right)=X$;

2. For any $j \in\{1,2,3,4\}, f_{\phi}\left(w^{(j)}\right)=w$,

Now, we observe $Z_{1}$. In the $\phi$-disc, there are some 1-cells with the labels $A, P, Q, \alpha$, $\beta, \gamma$, where we mean that a 1-cell is an open arcs. We call a 1-cell an edge. We denote the edges in the $\phi$-disc with the labels $A, P, Q, \alpha, \beta, \gamma$ by $A^{(i)}, P^{(i)}, Q^{(i)}, \alpha^{(j)}, \beta^{(j)}, \gamma^{(j)}$ respectively. Thus, the set $Z_{1}$ consists of fifteen edges $\left\{A^{(i)}, P^{(i)}, Q^{(i)}, \alpha^{(j)}, \beta^{(j)}, \gamma^{(j)} \mid i=\right.$ $1,2,3$ and $j=1,2\}$. Then, the map $\left.f_{\phi}\right|_{Z_{0}}$ is extended continuously on $Z_{0} \cup Z_{1}$ satisfying the following conditions.

1. For any $X \in\{A, P, Q\}$ and $i \in\{1,2,3\}, f_{\phi}\left(X^{(i)}\right)=X$;

2. For any $X \in\{\alpha, \beta, \gamma\}$ and $j \in\{1,2\}, f_{\phi}\left(X^{(j)}\right)=X$;

3. $f_{\phi}$ is a homeomorphism on each cell in $Z_{0} \cup Z_{1}$.

At last, we consider $Z_{2}$. Let $\rho_{+}, \sigma_{+}, \tau_{+}$be oriented 2-cells (open 2-discs) in the upper half disc of $D_{\phi}$ with the boundary $\partial\left(\operatorname{cl}\left(\rho_{+}\right)\right)=A, \partial\left(\operatorname{cl}\left(\sigma_{+}\right)\right)=P Q \bar{\alpha}, \partial\left(\operatorname{cl}\left(\tau_{+}\right)\right)=$ $A Q \bar{\gamma} P A \bar{P} \bar{\beta} \bar{Q}$ respectively, see Figure 2, where $\operatorname{cl}(X)$ means the closure of $X$. Similarly, let $\rho_{-}, \sigma_{-}, \tau_{-}$be oriented 2-cells in the under half disc in $D_{\phi}$ such that $\partial\left(\operatorname{cl}\left(\rho_{-}\right)\right)=A$, $\partial\left(\operatorname{cl}\left(\sigma_{-}\right)\right)=P Q \bar{\alpha}, \partial\left(\operatorname{cl}\left(\tau_{-}\right)\right)=A Q \bar{\gamma} P A \bar{P} \bar{\beta} \bar{Q}$ respectively. We call a 2-cell a face. Then, the set $Z_{2}$ consists of the faces $\left\{\rho_{+}, \rho_{-}, \sigma_{+}, \sigma_{-}, \tau_{+}, \tau_{-}, \operatorname{Int}\left(E_{\phi}\right)\right\}$. Then, the map $f_{\phi} \mid Z_{0} \cup Z_{1}$ is extended continuously on $Z_{0} \cup Z_{1} \cup Z_{2}\left(\cong S^{2}\right.$ ) satisfying the following conditions:

1. For any $X \in\{\rho, \sigma, \tau\}, f_{\phi}\left(X_{+}\right)=f_{\phi}\left(X_{-}\right)=X$;

2. $f_{\phi}$ is a homeomorphism on each cell in $Z_{0} \cup Z_{1} \cup Z_{2}$. Thereby, $f_{\phi}: S^{2} \rightarrow S^{2} / f_{\phi}$ is determined by the $\phi$-disc up to isotopy. We call the pasting information an identification map $f_{\phi}$ of the $\phi$ - disc.

Remark 2.1. Note that the label $X$ of each cell in $\phi$-disc is the name of the cell in $S^{2} / f_{\phi}$.

Let $G_{\phi}$ be the connected 3-regular graph with labels on $S^{2}$ associated with the underling space of $Z_{0} \cup Z_{1}$. Note that each of $S^{2} \backslash G_{\phi}$ is an open 2 - disc. Thus, $D_{\phi}, E_{\phi}, D_{\phi} / f_{\phi}$ and $E_{\phi} / f_{\phi}$ have cell structure. We call the triple $\left(S^{2}, G_{\phi}, f_{\phi}\right)$ as $\phi$-diagram.

Since $f_{\phi}$ is a map on $S^{2}\left(=\partial B^{3}\right)$, the quotient space $V_{\phi}:=B^{3} / f_{\phi}$ is defined. We can confirm that the quotient space $V_{\phi}$ is a compact 3-manifold by examining a neighborhood of each point of $B^{3}$. Note that the boundary of the 3-manifold $V_{\phi}$ is $E_{\phi} / f_{\phi}$, and the image $f_{\phi}\left(\partial D_{\phi}\right)=f_{\phi}\left(\partial E_{\phi}\right)$ is the theta-curve $\theta$ (Figure 1) embedded in $\partial V_{\phi}$ such that $\partial V_{\phi} \backslash \theta \cong$ $\operatorname{Int}\left(E_{\phi}\right)$. 


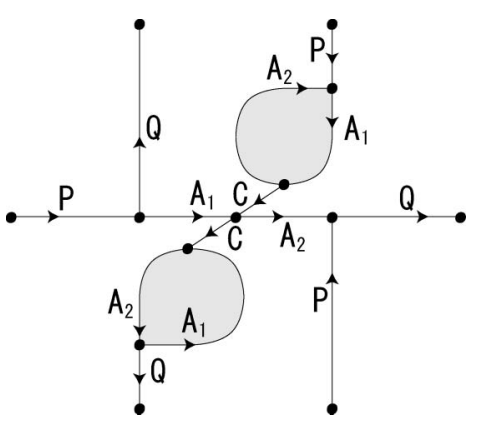

FIGURE 4. $D_{\phi}^{\prime}$.

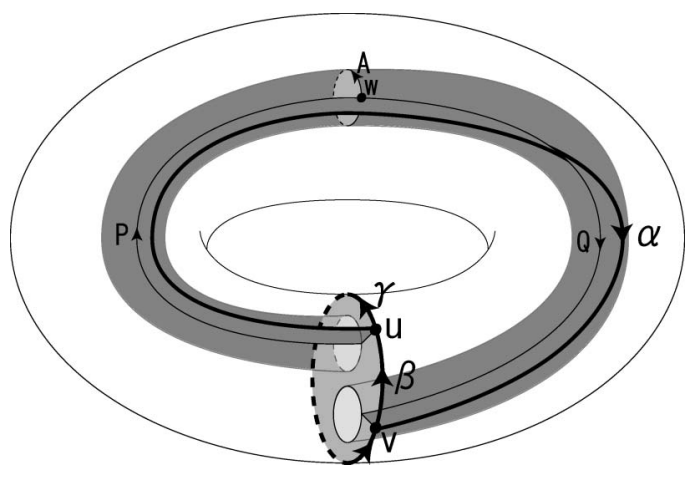

FIGURE 5. The solid torus $V_{\phi}$.

THEOREM 2.2. The manifold $V_{\phi}$ is homeomorphic to a solid torus.

Proof. By the definition of $f_{\phi}, E_{\phi} / f_{\phi}$ is the boundary of the manifold $V_{\phi}$, which is a free face of $V_{\phi}$. Thus, the manifold $V_{\phi}$ collapses to the cell complex $D_{\phi} / f_{\phi}$. And the cell complex $D_{\phi} / f_{\phi}$ collapses to the cell complex $D_{\phi}^{\prime} / f_{\phi}$, where $D_{\phi}^{\prime}$ is shown in Figure 4.

The cell complex $D_{\phi}^{\prime} / f_{\phi}$ collapses to the loop $C / f_{\phi}$, where $C$ is the edge shown in Figure 4. Thus, the manifold $V_{\phi}$ collapses to $S^{1}$.

Thereby, $V_{\phi}$ is homeomorphic to either a solid torus or a solid Klein bottle. By the definition of the map $f_{\phi}$, the manifold $V_{\phi}$ is orientable. Thus, it must be homeomorphic to a solid torus.

The solid torus $V_{\phi}$ is shown in Figure 5. The bold lines $\alpha, \beta$ and $\gamma$ are embedded in $\partial V_{\phi}$, and the others are embedded in $\operatorname{Int}\left(V_{\phi}\right)$. And shaded faces are $D_{\phi} / f_{\phi}$ embedded in $\operatorname{Int}\left(V_{\phi}\right)$.

2.2. The case $w(L, R) \neq \phi$. In the previous subsection, we observed the case when $w(L, R)$ is the empty word. In this subsection, we consider the case when $w(L, R)$ is not the empty word. For a word $w$, we will define $w$-disc $D_{w}$, and show that $B^{3} / f_{w}$ is homeomorphic to a solid torus, where $f_{w}$ is an identification map on $\partial B^{3}$ induced by $D_{w}$.

Now, we define the two discs $D_{w}$ and $D_{w}^{*}$ for a word $w$. Denote by $\mathcal{H}_{L}, \mathcal{H}_{\bar{L}}, \mathcal{H}_{R}, \mathcal{H}_{\bar{R}}$ the annuli shown in Figure 6, 7, 8, 9 respectively. At first, we consider the case that the length of the word $w$ is 1 , that is, $w=X$, where $X \in\{L, R, \bar{L}, \bar{R}\}$.

Definition 2.3. The disc $D_{X}^{*}$ is defined by the following steps.

Step 1. Rename the label $\mu$ of the vertices and the edges in $D_{\phi}$ into $\mu_{\phi}$, where $\mu=$ $u, v, w, A, P, Q, \alpha \beta, \gamma$.

Step 2. Rename the label $\mu$ of the vertices and the edges in $\mathcal{H}_{X}$ into $\mu_{X}$, where $\mu=$ $u, v, w, A, P, B, Q, \alpha \beta, \gamma$.

Step 3. Rename the label $\alpha^{\prime}, \beta^{\prime}$ and $\gamma^{\prime}$ of the edges in $\mathcal{H}_{X}$ into $\alpha_{\phi}, \beta_{\phi}$ and $\gamma_{\phi}$ respectively. 
Step 4. Glue the $\phi$-disc $D_{\phi}$ and the annulus $\mathcal{H}_{X}$ by identifying the corresponding circle $\alpha_{\phi} \beta_{\phi} \gamma_{\phi} \overline{\alpha_{\phi}} \overline{\beta_{\phi}} \overline{\gamma_{\phi}}$.

See, for example, Figure 10 for $D_{L}^{*}$.

Definition 2.4. The disc $D_{X}$ is obtained by the more two steps in addition to Definition 2.3.

Step 5. Delete the edges $\alpha_{\phi}, \beta_{\phi}$ and $\gamma_{\phi}$.

Step 6. Change the label $\mu_{\phi} v_{X}$ (or $v_{X} \mu_{\phi}$ ) into $v_{X}$. For example, in the case when $X=L$, there are arcs with label $Q_{\phi} B_{L}$, see Figure 10. Then, rename it into $B_{L}$. Similarly, change the label $A_{L} P_{\phi}$ into $A_{L}$.

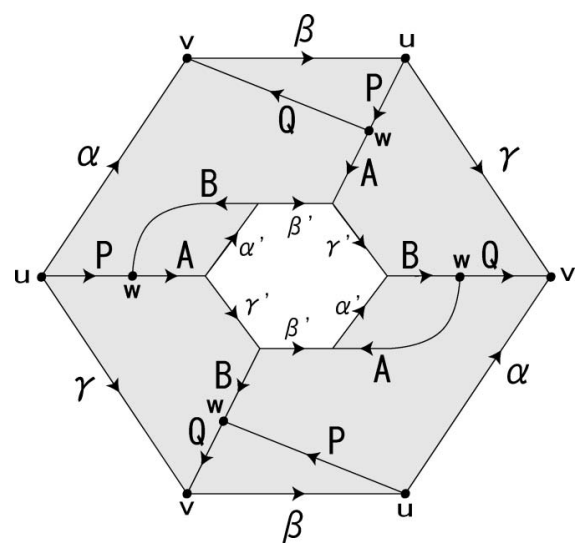

Figure 6. $\mathcal{H}_{L}$.

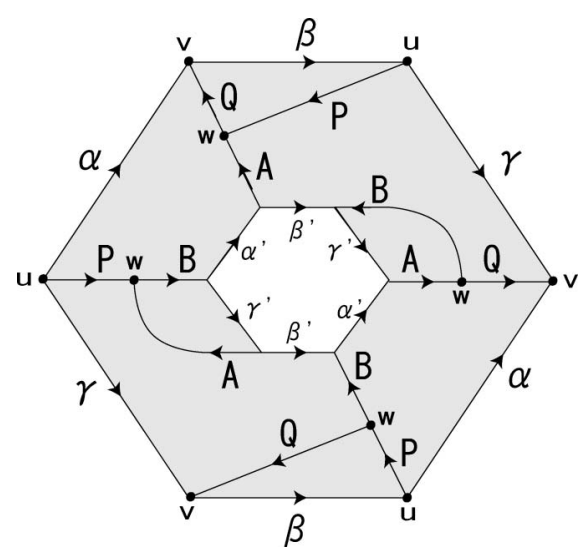

FIGURE 8. $\mathcal{H}_{R}$.

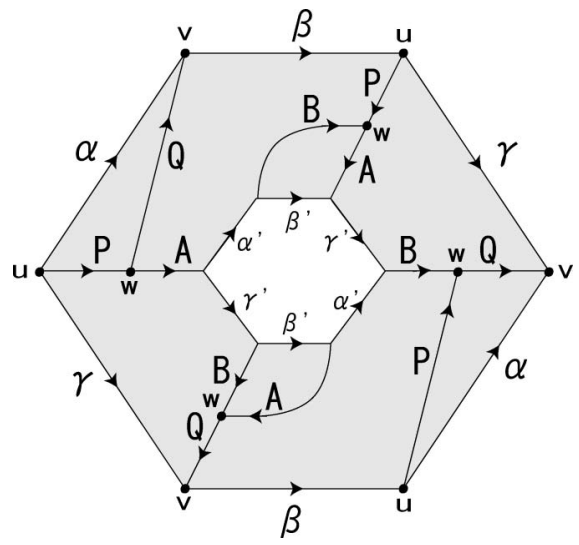

Figure 7. $\mathcal{H}_{\bar{L}}$.

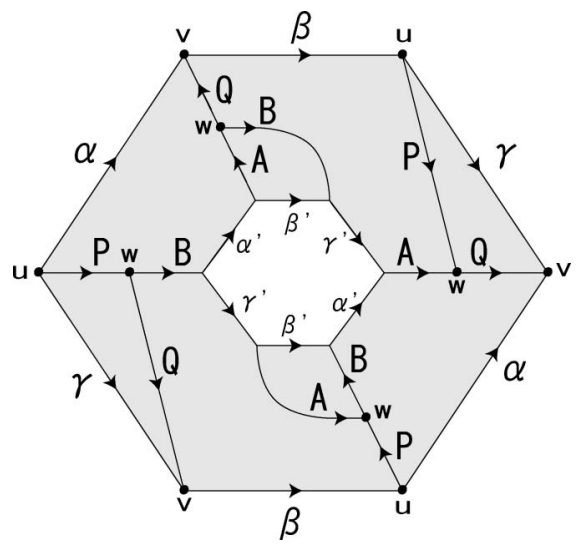

FIGURE $9 . \mathcal{H}_{\bar{R}}$. 
See, for example, Figure 11 for $D_{L}$.

Now, we define two discs $D_{w}^{*}$ and $D_{w}$ in the case when the length of the word $w$ is greater than 1. Our definition is given by the induction with respect to the length of the word $w$.

Suppose that two discs $D_{w}^{*}$ and $D_{w}$ are already defined for $w=X_{1} X_{2} \cdots X_{n}$, where $X_{i} \in\{L, R, \bar{L}, \bar{R}\}$. Then, let $w X$ be the word $X_{1} X_{2} \cdots X_{n} X$, where $X \in\{L, R, \bar{L}, \bar{R}\}$.

Definition 2.5. The disc $D_{w X}^{*}$ is defined by the following steps.

Step 1. Rename the label $\mu$ of the vertices and the edges in $\mathcal{H}_{X}$ into $\mu_{w X}$, where $\mu=$ $u, v, w, A, P, B, Q, \alpha \beta, \gamma$.

Step 2. Rename the label $\alpha^{\prime}, \beta^{\prime}$ and $\gamma^{\prime}$ of the edges in $\mathcal{H}_{X}$ into $\alpha_{w}, \beta_{w}$ and $\gamma_{w}$ respectively.

Step 3. Glue the disc $D_{w}^{*}$ and the annulus $\mathcal{H}_{X}$ by identifying the corresponding circle $\alpha_{w} \beta_{w} \gamma_{w} \overline{\alpha_{w}} \overline{\beta_{w}} \overline{\gamma_{w}}$.

DEFinition 2.6. The disc $D_{w X}$ is defined by the following steps.

Step 1. The same with Step 1 in Definition 2.5.

Step 2. The same with Step 2 in Definition 2.5.

Step 3. Glue the disc $D_{w}$ and the annulus $\mathcal{H}_{X}$ by identifying the corresponding circle $\alpha_{w} \beta_{w} \gamma_{w} \overline{\alpha_{w}} \overline{\beta_{w}} \overline{\gamma_{w}}$.

Step 4. Delete the edges $\alpha_{w}, \beta_{w}$ and $\gamma_{w}$.

Step 5. Change the label $\mu_{w} v_{w X}$ (or $v_{w X} \mu_{w}$ ) into $v_{w X}$.

Now, we consider an identification map $f_{w}$. Assume that the disc $D_{w}$ is embedded in $S^{2}$ (= $\left.\partial B^{3}\right)$ as the case $w=\phi$. Denote by $E_{w}$ the $\operatorname{disc} S^{2} \backslash \operatorname{Int}\left(D_{w}\right)$. As the case of $\phi$-disc, we can define an identification map $f_{w}: S^{2} \rightarrow S^{2} / f_{w}$ and a 3 - manifold $V_{w}=B^{3} / f_{w}$. Then,

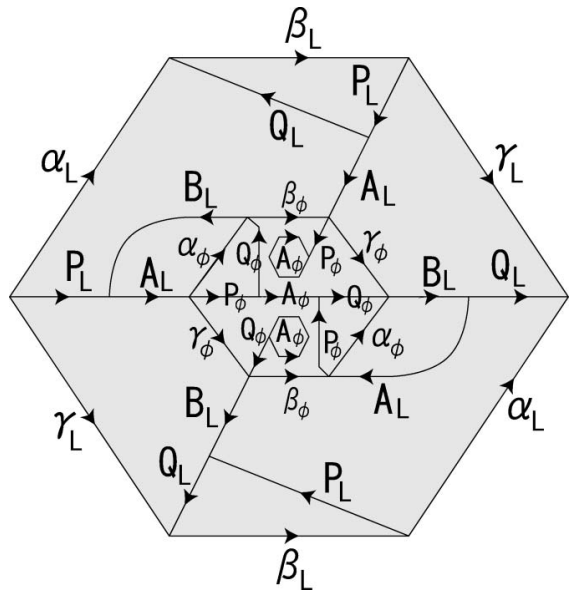

FiguRE $10 . \quad D_{L}^{*}$.

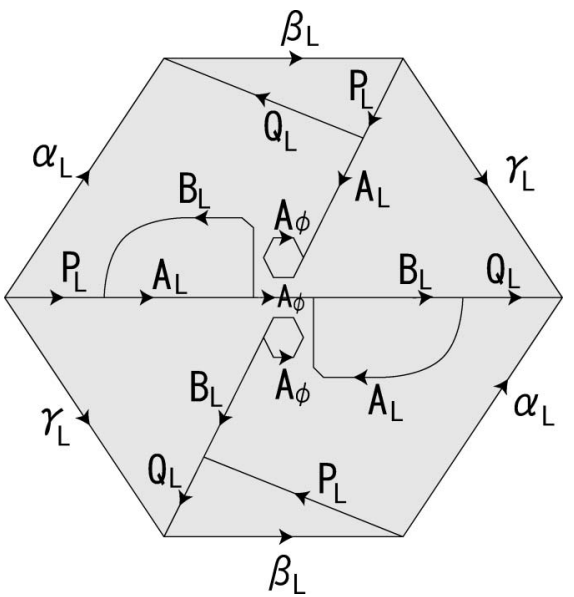

Figure $11 . D_{L}$. 
the image $f_{w}\left(\partial D_{w}\right)=f_{w}\left(\partial E_{w}\right)$ is the theta-curve $\theta$ (Figure 1) embedded in $\partial V_{w}$ such that $\partial V_{w} \backslash \theta \cong \operatorname{Int}\left(E_{w}\right)$.

THEOREM 2.7. The manifold $V_{w}$ is a homeomorphic to a solid torus.

Proof. At first, we prove that the manifold $V_{w}$ collapses to $S^{1}$ inductively. Since the manifold $V_{\phi}$ collapses to $S^{1}$ by the proof of Theorem 2.2, it is enough to show that if $V_{w}$ collapses to $S^{1}$ then $V_{w X}$ collapses to $S^{1}$ for each $X \in\{L, R, \bar{L}, \bar{R}\}$.

We consider the case $X=L$. Then, the manifold $V_{w L}$ collapses to the cell complex $D_{w L} / f_{w L}$ (Figure 12).

It collapses to the cell complex $D_{w L}^{\prime} / f_{w L}$ (Figure 13). Furthermore, the cell complex $D^{\prime}{ }_{w L} / f_{w L}$ collapses to the cell complex $D^{\prime \prime}{ }_{w L} / f_{w L}$, (Figure 14). At last, the cell complex $D^{\prime \prime}{ }_{w L} / f_{w L}$ collapses to the cell complex $D^{\prime \prime \prime}{ }_{w L} / f_{w L}$ (Figure 15). By the definition of $f_{w}$, the cell complex $D_{w L}^{\prime \prime \prime} / f_{w L}$ coincides with the cell complex $D_{w}^{\prime \prime \prime} / f_{w}$. By the assumption of induction, the cell complex $D_{w}^{\prime \prime \prime} / f_{w}$ collapses to $S^{1}$. So, $V_{w L}$ collapses to $S^{1}$. Thus, for any word $w$, the 3-manifold $V_{w}$ collapses to $S^{1}$. In the case $X=R, \bar{L}, \bar{R}$, we can prove similar to the case $X=L$. Since the manifold $V_{w}$ is orientable, we conclude that $V_{w}$ is homeomorphic to a solid torus.

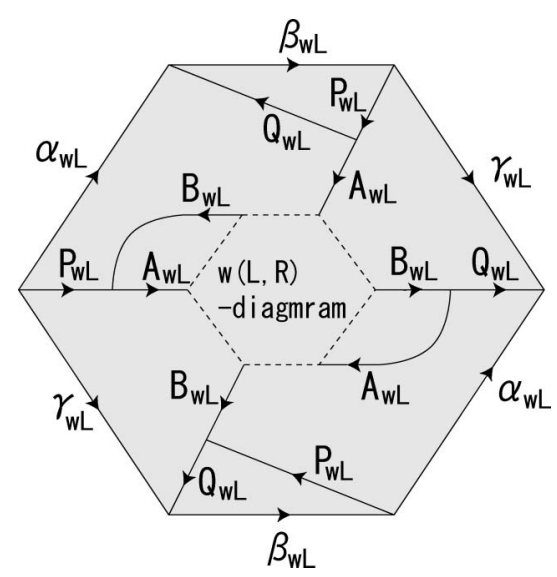

FIGURE 12. $D_{L}^{*}$.

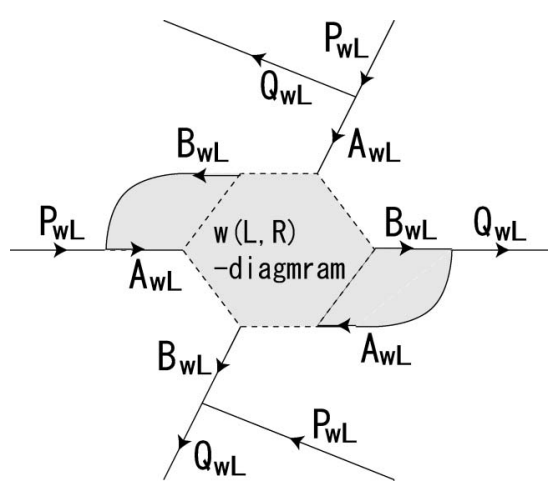

FIGURE 13. $D_{w L}^{\prime}$. 


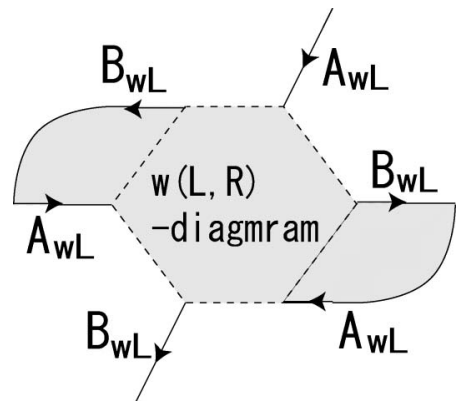

FIGURE 14. $D_{w(L, R) L}^{\prime \prime}$

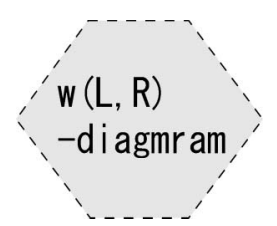

FIGURE $15 . \quad D_{w(L, R) L}^{\prime \prime \prime}$

\section{Meridian, longitude and fiber structure of $V_{w(L, R)}$}

In this section, we consider a meridian-longitude system $\left(m_{w}, l_{w}\right)$ of the solid torus $V_{w}$, where a meridian-longitude system $\left(m_{w}, l_{w}\right)$ is a pair of loops $m_{w}$ and $l_{w}$ satisfying the following conditions:

1. $\quad m_{w}$ and $l_{w}$ are essential loops embedded in $\partial V_{w}$

2. $m_{w}$ and $l_{w}$ intersect at a point.

3. $\ll m_{w} \gg=1$ and $\ll l_{w} \gg \neq 1$ in $\pi_{1}\left(V_{w}\right)$, where $\ll \gg$ is a homotopy class.

In $\S 3.1$, we give a presentation of loops embedded in $\partial V_{w}$, that is, we define two generators $\ll x_{w} \gg$ and $\ll y_{w} \gg$ of $\pi_{1}\left(\partial V_{w}\right)$ for each word $w$. Then, any loop embedded in $\partial V_{w}$ is presented by an element $a_{w}\left[x_{w}\right]+b_{w}\left[y_{w}\right]$ in $H_{1}\left(\partial V_{w}\right)$ uniquely, where $a_{w}$ and $b_{w}$ are coprime integers and [ ] is the homology class. In $\S 3.2$ and $\S 3.3$, we consider the coefficients $a_{w}$ and $b_{w}$ of the meridian and a longitude of $V_{w}$.

3.1. Loops embedded in $\partial V_{w(L, R)}$. In $\S 2$, we showed that $E_{w} / f_{w}$ is the boundary of the solid torus $V_{w}$. Thus, three loops $\overline{\alpha_{w} \beta_{w}}, \gamma_{w} \beta_{w}$ and $\gamma_{w} \overline{\alpha_{w}}$ are embedded in $\partial V_{w}$. We denote them by $x_{w}, y_{w}$ and $z_{w}$ respectively.

Proposition 3.1. For any word $w$, the two elements $\ll x_{w} \gg$ and $\left\langle y_{w} \gg\right.$ forms a basis of the fundamental group $\pi_{1}\left(\partial V_{w}\right)$, where $\left\langle x_{w} \gg\right.$ and $\left\langle y_{w} \gg\right.$ are the homotopy classes of the loops $x_{w}$ and $y_{w}$ respectively.

Proof. Consider the loop $A_{w}$ embedded in $\partial V_{w}$, where the loop $A_{w}$ is shown in Figure 16.

Two loops $A_{w}$ and $\gamma_{w} \beta_{w}$ are homotopic in $\partial V_{w}$. By the method of cut and paste, we know two loops $A_{w}$ and $\overline{\alpha_{w} \beta_{w}}$ are essential loops and intersect at one point in $\partial V_{w}$.

By Proposition 3.1, any loop embedded in $\partial V_{w}$ is presented by an element $a_{w}\left[x_{w}\right]+$ $b_{w}\left[y_{w}\right]$ in $H_{1}\left(\partial V_{w}\right)$ uniquely, where $a_{w}$ and $b_{w}$ is coprime integers.

3.2. The case $w(L, R)=\phi$. In this subsection, we consider a meridian-longitude system of the solid torus $V_{\phi}$. 


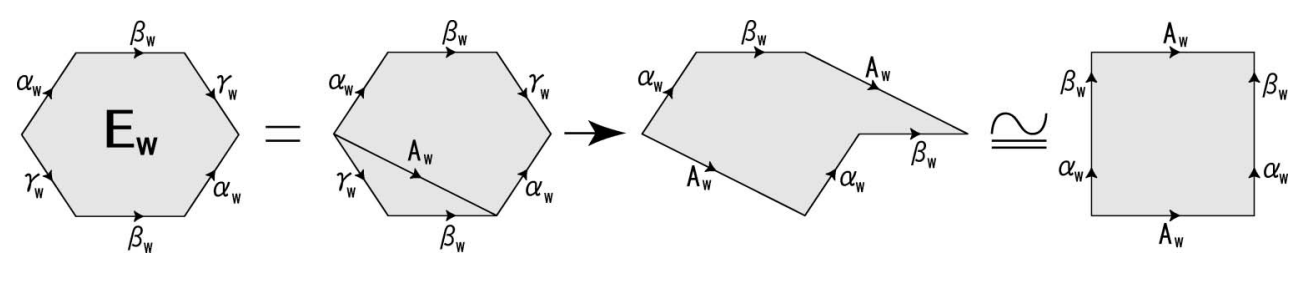

FIGURE 16. $E_{w}$.

THEOREM 3.2. A pair of the loops $\left(y_{\phi}, x_{\phi}\right)$ is a meridian-longitude system of the solid torus $V_{\phi}$.

PROOF. According to the proof of Proposition 3.1, loops $x_{\phi}$ and $y_{\phi}$ are essential loops in $\partial V_{\phi}$. Furthermore, we can regard $x_{\phi}$ and $y_{\phi}$ as loops intersecting at a point with the argument of general position. Thus, all we have to do is to show that $\ll x_{\phi} \gg \neq 1$ and $\ll y_{\phi} \gg=1$ in $\pi_{1}\left(V_{\phi}\right)$. We consider the fundamental group $\pi_{1}\left(V_{\phi}\right)$. Since $V_{\phi}$ collapses to $D_{\phi} / f_{\phi}$, the fundamental group $\pi_{1}\left(V_{\phi}\right)$ is isomorphic to $\pi_{1}\left(D_{\phi} / f_{\phi}\right)$. So, we consider $\pi_{1}\left(D_{\phi} / f_{\phi}\right)$. We choose a base point of $D_{\phi} / f_{\phi}$ as the vertex $u=f_{\phi}\left(u^{(i)}\right)$, see Figure 2 .

The fundamental group $\pi_{1}\left(D_{\phi} / f_{\phi}\right)$ is obtained by the following two steps. First, we consider a maximal tree $Q \beta$ of $G_{\phi}$, where $G_{\phi}:=\left|Z_{0} \cup Z_{1}\right|$ and $Z_{0}$ and $Z_{1}$ was defined in $\S 2.1$. Then, any loop embedded in $D_{\phi} / f_{\phi}$ is homotopic to some loop which is a finite product of the loops $\alpha \beta, \gamma \beta, P Q \beta$ and $\bar{\beta} \bar{Q} A Q \beta$. That is, $\pi_{1}\left(D_{\phi} / f_{\phi}\right)$ is generated by the homotopy classes of the loops $\alpha \beta, \gamma \beta, P Q \beta$ and $\bar{\beta} \bar{Q} A Q \beta$. We denote them by $\hat{\alpha}, \hat{\gamma}, \hat{P}, \hat{A}$ respectively. And, any relator of $\pi_{1}\left(D_{\phi} / f_{\phi}\right)$ is a sequence of $\left\{\hat{P} \hat{\alpha}^{-1}, \hat{A} \hat{\gamma}^{-1}, \hat{A}\right\}$ which are homotopy classes of the loops each of which bounds a face of $D_{\phi} / f_{\phi}$. Thus, we get

$$
\begin{aligned}
\pi_{1}\left(V_{\phi}\right) & =\pi_{1}\left(D_{\phi} / f_{\phi}, u\right) \\
& =<\hat{\alpha}, \hat{\gamma}, \hat{P}, \hat{A} \mid \hat{P} \hat{\alpha}^{-1}, \hat{A} \hat{\gamma}^{-1}, \hat{A}> \\
& =<\hat{\alpha}, \hat{\gamma}, \hat{P} \mid \hat{P} \hat{\alpha}^{-1}, \hat{\gamma}^{-1}> \\
& =<\hat{\alpha}, \hat{P} \mid \hat{P} \hat{\alpha}^{-1}> \\
& =<\hat{\alpha} \mid->.
\end{aligned}
$$

And the generator of $\pi_{1}\left(V_{\phi}\right)$ is $\hat{\alpha}$. Since $\hat{\alpha}$ is the homotopy class of the loop $x_{\phi}=\overline{\alpha_{\phi} \beta_{\phi}}$, we get $\ll x_{\phi} \gg \neq 1$. And $\hat{\gamma}^{-1}$ is a relator of $\pi_{1}\left(V_{\phi}\right)$. Since $\hat{\gamma}$ is the homotopy class of a loop $y_{\phi}=\gamma_{\phi} \beta_{\phi}$, we have $\ll y_{\phi} \gg=1$.

Now, we consider the loop $z_{\phi}=\gamma_{\phi} \overline{\alpha_{\phi}}$ in $\partial V_{\phi}$.

DEFINITION 3.3. Let $V$ be a solid torus with the meridian-longitude system $(m, l)$. Then, a loop $\lambda$ in $\partial V$ is the $(p, q)$ - type if the condition $[\lambda]=p[l]+q[m]$ holds in $H_{1}(\partial V)$. 
Then, we consider the type of the loop $z_{\phi}$ for a solid torus $V_{\phi}$ with the meridian-longitude system $\left(y_{\phi}, x_{\phi}\right)$ given in Theorem 3.2.

THEOREM 3.4. The loop $z_{\phi}$ is the $(1,1)$-type.

Proof. By the definition, the homotopy class of the loops $x_{\phi}, y_{\phi}$ and $x_{\phi}$ satisfy the following condition in $\pi_{1}\left(\partial V_{\phi}\right)$.

$$
\begin{aligned}
\ll z_{\phi} \gg & =\ll \gamma_{\phi} \overline{\alpha_{\phi}} \gg \\
& =\ll \gamma_{\phi} \beta_{\phi} \overline{\beta_{\phi}} \overline{\alpha_{\phi}} \gg \\
& =\ll \gamma_{\phi} \beta_{\phi} \gg \ll \overline{\alpha_{\phi} \beta_{\phi}} \gg .
\end{aligned}
$$

Thus, the loop $z_{\phi}$ satisfies the condition $\left[z_{\phi}\right]=\left[x_{\phi}\right]+\left[y_{\phi}\right]$ in $H_{1}\left(\partial V_{\phi}\right)$.

3.3. The case $w(L, R)$ is not the empty. In this subsection, we consider a meridianlongitude system of the solid torus $V_{w}$ is the case when the word $w$ is not the empty word. In $\S 3.3 .1$, we give two theorems about meridian-longitude systems of the solid torus $V_{w}$. In $\S 3.3 .2$, we prepare lemmas. In $\S 3.3 .3$, we give the proofs of the theorems.

3.3.1. Theorems. For a word $w=X_{1} X_{2} \ldots X_{n}$, where $X_{i} \in\{L, R, \bar{L}, \bar{R}\}$, we define the matrix $M_{w}=U_{X_{1}} U_{X_{2}} \cdots U_{X_{n}}$, where $U_{L}=\left(\begin{array}{ll}1 & 1 \\ 0 & 1\end{array}\right), U_{R}=\left(\begin{array}{ll}1 & 0 \\ 1 & 1\end{array}\right), U_{\bar{L}}=U_{L}^{-1}$ and $U_{\bar{R}}=U_{R}{ }^{-1}$.

THEOREM 3.5. There is a meridian-longitude system $\left(m_{w}, l_{w}\right)$ of $V_{w}$ satisfying the following equations in $H_{1}\left(\partial V_{w}\right)$ :

$$
\begin{aligned}
{\left[m_{w}\right] } & =-M_{1,2}\left[x_{w}\right]+M_{1,1}\left[y_{w}\right] ; \\
{\left[l_{w}\right] } & =M_{2,2}\left[x_{w}\right]-M_{2,1}\left[y_{w}\right],
\end{aligned}
$$

where $M_{i, j}$ is the $(i, j)$-element of the matrix $M_{w}$.

Now, we will see another theorem. We use the following notation for the expansion into continued fraction:

$$
\left[a_{1}, a_{2}, \cdots, a_{n-1}, a_{n}\right]:=\frac{1}{a_{1}+\frac{1}{a_{2}+\frac{1}{\ddots+\frac{1}{a_{n}}}}} .
$$

For a pair of natural numbers $p, q$ such that $p>q$, we define an alternative word $A(p, q)$ as follows:

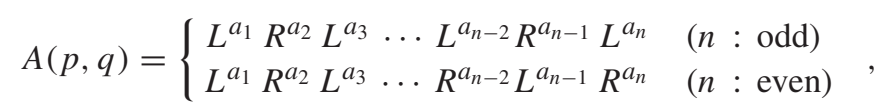


where the natural numbers $a_{i}$ are defined by $q / p=\left[a_{1}, a_{2}, \cdots, a_{n}, 1\right]$.

THEOREM 3.6. For the solid torus $V_{A(p, q)}$ with the meridian-longitude system $\left(m_{A(p, q)}, l_{A(p, q)}\right)$ given by Theorem 3.5 , the loop $z_{A(p, q)}$ is the $(p, q)$-type.

Notation 3.7. We call $V_{A(p, q)}$ as $(p, q)$-type solid torus and denote it by $V_{p, q}$.

3.3.2. Lemmas. In this subsection, we show two lemmas. The first one is concerned with the fundamental group of the solid torus $V_{w}$. The second one is the calculation of the matrices $U_{L}, U_{R}, U_{\bar{L}}$ and $U_{\bar{R}}$.

Now, we see the first one. At first, we consider the solid torus $V_{L}$, where $V_{L}$ is the solid torus $V_{w}$ in the case $w=L$. Since the solid torus $V_{L}$ collapses to $D_{L} / f_{L}$, the fundamental group $\pi_{1}\left(V_{L}\right)$ is isomorphic to $\pi_{1}\left(D_{L} / f_{L}\right)$. By the definition, the fundamental group $\pi_{1}\left(D_{L} / f_{L}\right)$ is isomorphic to $\pi_{1}\left(D_{L}^{*} / f_{L}\right)$. Thereby, the fundamental group $\pi_{1}\left(V_{L}\right)$ is isomorphic to $\pi_{1}\left(D_{L}^{*} / f_{L}\right)$.

Thus, we consider $\pi_{1}\left(D_{L}^{*} / f_{L}\right)$. We take a base point $u_{L}$ of $D_{L}^{*} / f_{L}$ and a maximal tree $\beta_{L} P_{L} A_{L} \beta_{\phi} P_{\phi}$ of $D_{L}^{*} / f_{L}$, see Figure 10 , where $\beta_{L} P_{L} A_{L} \beta_{\phi} P_{\phi}$ means the union of the edges $\beta_{L}, P_{L}, A_{L}, \beta_{\phi}$ and $P_{\phi}$. And we consider the generators and relators similar to the case $w=\phi$. Also we use the same notations $\hat{X}$ in $\S 3$.2. For convenience, we denote $X_{L}$ and $X_{\phi}$ by $X$ and $X^{\prime}$ respectively. Then, we have

$$
\begin{aligned}
& \pi_{1}\left(V_{L}\right) \\
& =\pi_{1}\left(D_{L}^{*} / f_{L}, u_{L}\right) \\
& =<\hat{\alpha}^{\prime}, \hat{\gamma}^{\prime}, \hat{Q}^{\prime}, \hat{A}^{\prime}, \hat{B}^{-1}, \hat{Q}, \hat{\alpha}, \hat{\gamma} \\
& \mid \hat{Q}^{\prime} \hat{\alpha}^{\prime-1}, \hat{Q}^{\prime-1} \hat{A}^{\prime} \hat{Q}^{\prime} \hat{\gamma}^{\prime}{ }^{-1} \hat{A}^{\prime}, \hat{A}^{\prime}, \hat{\alpha^{\prime}} \hat{B}, \hat{Q} \hat{\alpha}^{-1} \hat{B}^{-1}, \hat{Q}, \hat{\gamma^{\prime}} \hat{B} \hat{Q} \hat{\gamma}^{-1}> \\
& =<\hat{\alpha}^{\prime}, \hat{\gamma}^{\prime}, \hat{Q}^{\prime}, \hat{B}^{-1}, \hat{Q}, \hat{\alpha}, \hat{\gamma} \mid \hat{Q}^{\prime} \hat{\alpha}^{\prime}, \hat{\gamma}^{\prime-1}, \hat{\alpha^{\prime}} \hat{B}, \hat{Q} \hat{\alpha}^{-1} \hat{B}^{-1}, \hat{Q}, \hat{\gamma}^{\prime} \hat{B} \hat{Q} \hat{\gamma}^{-1}> \\
& =<\hat{\alpha}^{\prime}, \hat{\gamma}^{\prime}, \hat{B}^{-1}, \hat{Q}, \hat{\alpha}, \hat{\gamma} \mid \hat{\gamma^{\prime}}, \hat{\alpha^{\prime}} \hat{B}, \hat{Q} \hat{\alpha}^{-1} \hat{B}^{-1}, \hat{Q}, \hat{\gamma^{\prime}} \hat{B} \hat{Q} \hat{\gamma}^{-1}> \\
& =<\hat{\alpha^{\prime}}, \hat{\gamma^{\prime}}, \hat{Q}, \hat{\alpha}, \hat{\gamma} \mid \hat{\gamma^{\prime}}, \hat{Q} \hat{\alpha}^{-1} \hat{\alpha^{\prime}}, \hat{Q}, \hat{\gamma}^{\prime} \hat{\alpha}^{-1} \hat{Q} \hat{\gamma}^{-1}> \\
& =<\hat{\alpha}^{\prime}, \hat{\gamma^{\prime}}, \hat{\alpha}, \hat{\gamma} \mid \hat{\gamma}^{\prime}, \hat{\alpha}^{-1} \hat{\alpha}^{\prime}, \hat{\gamma}^{\prime} \hat{\alpha}^{-1} \hat{\gamma}^{-1}> \\
& =<\hat{\alpha}^{\prime} \mid->\text {. }
\end{aligned}
$$

And we get the two relations $\hat{\alpha}^{-1} \hat{\alpha}^{\prime}=1$ and $\hat{\gamma}^{\prime}{\hat{\alpha^{\prime}}}^{-1} \hat{\gamma}^{-1}=1$ in $\pi_{1}\left(V_{L}\right)$. Since, the homology class of the loops $x_{\phi}, y_{\phi}, x_{L}$ and $y_{L}$ are ${\hat{\alpha^{\prime}}}^{-1}, \hat{\gamma}^{\prime}, \hat{\alpha}^{-1}$ and $\hat{\gamma}$ respectively, we get the following relations in $H_{1}\left(V_{L}\right)$ :

$$
\left\{\begin{array}{l}
{\left[x_{L}\right]=\left[x_{\phi}\right]} \\
{\left[y_{L}\right]=\left[x_{\phi}\right]+\left[y_{\phi}\right]}
\end{array} \Longleftrightarrow{ }^{t}\left(\begin{array}{l}
{\left[x_{L}\right]} \\
{\left[y_{L}\right]}
\end{array}\right)=t^{t}\left(\begin{array}{l}
{\left[x_{\phi}\right]} \\
{\left[y_{\phi}\right]}
\end{array}\right) U_{L},\right.
$$


where ${ }^{t}(\quad)$ means the transposition and [ ] means the homology class and $U_{L}$ is defined in $\S 3.3 .1$. Since the generator of $\pi_{1}\left(V_{L}\right)$ is $\hat{\alpha^{\prime}}$, we get $\left[x_{\phi}\right] \neq 0$ in $H_{1}\left(V_{L}\right)$. And by the relation of $\pi_{1}\left(V_{L}\right)$, we have $\left[y_{\phi}\right]=0$ in $H_{1}\left(V_{L}\right)$.

In the case $X=\bar{L}, R, \bar{R}$, we take a base point $u_{X}$ of $\pi_{1}\left(D_{X}^{*} / f_{X}\right)$ and a maximal tree $\beta_{\bar{L}} P_{\bar{L}} A_{\bar{L}} \beta_{\phi} P_{\phi}, \beta_{R} Q_{R} A_{R} \beta_{\phi} P_{\phi}$ and $\beta_{\bar{R}} Q_{\bar{R}} A_{\bar{R}} \beta_{\phi} P_{\phi}$ respectively. Then, we get the relations

$\left(\begin{array}{l}{\left[x_{X}\right]} \\ {\left[y_{X}\right]}\end{array}\right)={ }^{t}\left(\begin{array}{l}{\left[x_{\phi}\right]} \\ {\left[y_{\phi}\right]}\end{array}\right) U_{X},\left[x_{\phi}\right] \neq 0$ and $\left[y_{\phi}\right]=0$ in $H_{1}\left(V_{X}\right)$, where the matrix $U_{\bar{L}}, U_{R}$ and $U_{\bar{R}}$ are defined in $\S 3.3 .1$.

At last, for any word $w=X_{1} X_{2} \cdots X_{n}$, where $X_{i} \in\{L, R, \bar{L}, \bar{R}\}$, we consider $H_{1}\left(V_{w}\right)$. We take a base point $u_{w}$ of $D_{w}^{*} / f_{w}$ and a maximal tree $\left(\beta_{\phi} D_{\phi}\right) \cup\left(\coprod_{i=1}^{n} m_{i}\right)$, where $m_{i}=$ $\left\{\begin{array}{ll}\beta_{X_{i}} P_{X_{i}} A_{X_{i}} & \left(X_{i}=L, \bar{L}\right) ; \\ \beta_{X_{i}} Q_{X_{i}} A_{X_{i}} & \left(X_{i}=R, \bar{R}\right) .\end{array}\right.$ Then, we get the equation ${ }^{t}\left(\begin{array}{c}{\left[x_{w X}\right]} \\ {\left[y_{w X}\right]}\end{array}\right)={ }^{t}\left(\begin{array}{l}{\left[x_{w}\right]} \\ {\left[y_{w}\right]}\end{array}\right) U_{X}$ for any $X=L, R, \bar{L}, \bar{R}$. Thus, we have the following lemma.

LEMMA 3.8. The following two relations 1 and 2 hold in $H_{1}\left(V_{w}\right)$, where the loops $x_{w}$ and $y_{w}$ are embedded in $\partial V_{w}$ and the loops $x_{\phi}$ and $y_{\phi}$ are embedded in $\operatorname{Int}\left(V_{w}\right)$.

1. ${ }^{t}\left(\begin{array}{l}{\left[x_{w}\right]} \\ {\left[y_{w}\right]}\end{array}\right)={ }^{t}\left(\begin{array}{l}{\left[x_{\phi}\right]} \\ {\left[y_{\phi}\right]}\end{array}\right) M_{w}$, where the matrix $M_{w}$ is defined in Theorem 3.5 and $[$ ] means a homology class.

2. $\left[x_{\phi}\right] \neq 0$ and $\left[y_{\phi}\right]=0$.

Now, we observe another lemma. For $n$ natural numbers $a_{i}(1 \leq i \leq n)$, two matrices $\mathcal{A}$ and $\mathcal{B}$ are defined as follows:

$$
\begin{aligned}
& \mathcal{A}=\left\{\begin{array}{lllll}
U_{L}^{a_{1}} & U_{R}^{a_{2}} U_{L}^{a_{3}} \cdots & U_{L}^{a_{n-2}} U_{R}^{a_{n-1}} U_{L}^{a_{n}} & (n: \text { odd }) \\
U_{L}^{a_{1}} U_{R}^{a_{2}} U_{L}^{a_{3}} \cdots & U_{R}^{a_{n-2}} U_{L}^{a_{n-1}} U_{R}^{a_{n}} & (n: \text { even })
\end{array},\right. \\
& \mathcal{B}=\left\{\begin{array}{llll}
U_{R}^{a_{1}} U_{L}^{a_{2}} U_{R}^{a_{3}} \cdots & U_{R}^{a_{n-2}} U_{L}^{a_{n-1}} U_{R}^{a_{n}} & (n: \text { odd }) \\
U_{R}^{a_{1}} U_{L}^{a_{2}} U_{R}^{a_{3}} & \cdots & U_{L}^{a_{n-2}} U_{R}^{a_{n-1}} U_{L}^{a_{n}} & (n \text { : even })
\end{array} .\right.
\end{aligned}
$$

We denote by $A_{i, j}$ and $B_{i, j}$ the $(i, j)$-element of the matrix $\mathcal{A}$ and $\mathcal{B}$ respectively.

LEMMA 3.9. Two matrices $\mathcal{A}$ and $\mathcal{B}$ satisfy the following conditions:

1. $\frac{A_{2,1}+A_{2,2}}{A_{1,1}+A_{1,2}}=\frac{B_{1,1}+B_{1,2}}{B_{2,1}+B_{2,2}}=\left[a_{1}, a_{2}, \cdots, a_{n}, 1\right]$, where [ ] means the expansion into continued fraction defined in $\$ 3.3 .1$;

2. (a) Two natural numbers $A_{1,1}$ and $A_{1,2}$ are coprime, and two non-negative integers $A_{2,1}$ and $A_{2,2}$ are coprime.

(b) Two natural numbers $A_{1,1}+A_{1,2}$ and $A_{2,1}+A_{2,2}$ are coprime;

PROOF.

1. Let us consider a proof by induction on the length of the matrix $\mathcal{A}$ and $\mathcal{B}$, where the length is the natural number $n$ in the matrix $U_{X_{1}}^{a_{1}} U_{X_{2}}^{a_{2}} U_{X_{3}}^{a_{3}} \cdots U_{X_{n}}^{a_{n}}$. 
If $n=1$, then $\mathcal{A}=\left(\begin{array}{cc}1 & a_{1} \\ 0 & 1\end{array}\right)$ and $\mathcal{B}=\left(\begin{array}{cc}1 & 0 \\ a_{1} & 1\end{array}\right)$. So, we have $\frac{A_{2,1}+A_{2,2}}{A_{1,1}+A_{1,2}}=$ $\frac{B_{1,1}+B_{1,2}}{B_{2,1}+B_{2,2}}=\frac{1}{a_{1}+1}=\left[a_{1}, 1\right]$. Now, assume that the statement holds in the case $n-1$. Then, we will consider the case $n$.

(i) In the case $n$ is odd

$$
\begin{aligned}
\mathcal{A} & =U_{L}^{a_{1}} U_{R}^{a_{2}} U_{L}^{a_{3}} \cdots U_{L}^{a_{n}}=U_{L}^{a_{1}} \mathcal{B} \\
& =\left(\begin{array}{cc}
1 & a_{1} \\
0 & 1
\end{array}\right)\left(\begin{array}{cc}
B_{1,1} & B_{1,2} \\
B_{2,1} & B_{2,2}
\end{array}\right) \\
& =\left(\begin{array}{cc}
B_{1,1}+a_{1} B_{2,1} & B_{1,2}+a_{1} B_{2,2} \\
B_{2,1} & B_{2,2}
\end{array}\right),
\end{aligned}
$$

where $\mathcal{B}$ is the matrix with respect to $n-1$ natural numbers $a_{2}, a_{3}, \cdots, a_{n}$, that is, $\mathcal{B}=U_{R}^{a_{2}} U_{L}^{a_{3}} \cdots U_{L}^{a_{n}}$. Then, we obtain

$$
\begin{aligned}
\frac{A_{2,1}+A_{2,2}}{A_{1,1}+A_{1,2}} & =\frac{B_{2,1}+B_{2,2}}{B_{1,1}+a_{1} B_{2,1}+\left(B_{1,2}+a_{1} B_{2,2}\right)} \\
& =\frac{1}{a_{1}+\frac{B_{1,1}+B_{1,2}}{B_{2,1}+B_{2,2}}} .
\end{aligned}
$$

By the assumption of the induction, we have the following equation about the matrix $\mathcal{B}$.

$$
\frac{B_{1,1}+B_{1,2}}{B_{2,1}+B_{2,2}}=\left[a_{2}, a_{3}, \cdots, a_{n}, 1\right]
$$

Thus, we have

$$
\frac{1}{a_{1}+\frac{B_{1,1}+B_{1,2}}{B_{2,1}+B_{2,2}}}=\left[a_{1}, a_{2}, \cdots, a_{n}, 1\right] .
$$

Also, we have that

$$
\begin{aligned}
\mathcal{B} & =U_{R}^{a_{1}} U_{L}^{a_{2}} U_{R}^{a_{3}} \cdots U_{R}^{a_{n}}=U_{R}^{a_{1}} \mathcal{A} \\
& =\left(\begin{array}{cc}
1 & 0 \\
a_{1} & 1
\end{array}\right)\left(\begin{array}{ll}
A_{1,1} & A_{1,2} \\
A_{2,1} & A_{2,2}
\end{array}\right) \\
& =\left(\begin{array}{cc}
A_{1,1} & A_{1,2} \\
a_{1} A_{1,1}+A_{2,1} & a_{1} A_{1,2}+A_{2,2}
\end{array}\right)
\end{aligned}
$$

and

$$
\frac{B_{1,1}+B_{1,2}}{B_{2,1}+B_{2,2}}=\frac{A_{1,1}+A_{1,2}}{a_{1} A_{1,1}+A_{2,1}+\left(a_{1} A_{1,2}+A_{2,2}\right)}
$$




$$
=\frac{1}{a_{1}+\frac{A_{2,1}+A_{2,2}}{A_{1,1}+A_{1,2}}}=\left[a_{1}, a_{2}, \cdots, a_{n}, 1\right] .
$$

(ii) In the case $n$ is even

We can prove similar to the case (i).

2. (a) We have $\operatorname{det} U_{X}=1$ for all $X \in\{L, R, \bar{L}, \bar{R}\}$ by the definition of the matrix $U_{X}$. Since the property of the determinant: $\operatorname{det} A B=\operatorname{det} A \operatorname{det} B$, we get $\operatorname{det} \mathcal{A}=1$. It completes the proof.

2. (b) By the property of the determinant, we have $\operatorname{det} \mathcal{A}=\operatorname{det}\left(\begin{array}{ll}A_{1,1}+A_{1,2} & A_{1,2} \\ A_{2,1}+A_{2,2} & A_{2,2}\end{array}\right)$. Since $A_{1,1}+A_{1,2}, A_{1,2}, A_{2,1}+A_{2,2}$ and $A_{2,2}$ are natural numbers and $\operatorname{det} \mathcal{A}=1$, we get $A_{1,1}+A_{1,2}$ and $A_{2,1}+A_{2,2}$ are coprime.

\subsubsection{Proof of main theorems.}

PROOF OF THEOREM 3.5. There is a representative $y$ of homology class $\left[y_{w}\right]$ such that $y$ is an essential loop on a solid torus $V_{w}$ and $y$ intersect with $x_{w}$ at one point each other, where $x_{w}$ and $y_{w}$ are defined in $\S 3.1$. Since $\operatorname{det} M_{w}=1$ and two non-negative integers $M_{i, 1}$ and $M_{i, 2}$ are coprime for $i=1,2$, there are two essential loops $m_{w}$ and $l_{w}$ on $\partial V_{w}$ with one common point such that

$$
\left[m_{w}\right]=-M_{1,2}\left[x_{w}\right]+M_{1,1}\left[y_{w}\right] ; \quad\left[l_{w}\right]=M_{2,2}\left[x_{w}\right]-M_{2,1}\left[y_{w}\right] .
$$

Thus, the rest of the proof is to show $\left[l_{w}\right] \neq 0$ and $\left[m_{w}\right]=0$ in $H_{1}\left(V_{w}\right)$. By Lemma 3.8, we get the following equation in $H_{1}\left(V_{w}\right)$ :

$$
\begin{aligned}
& { }^{t}\left(\begin{array}{c}
{\left[x_{w}\right]} \\
{\left[y_{w}\right]}
\end{array}\right)={ }^{t}\left(\begin{array}{c}
{\left[x_{\phi}\right]} \\
{\left[y_{\phi}\right]}
\end{array}\right) M_{w} \\
& \Longleftrightarrow{ }^{t}\left(\begin{array}{c}
{\left[x_{w}\right]} \\
{\left[y_{w}\right]}
\end{array}\right)\left(\begin{array}{cc}
M_{2,2} & -M_{1,2} \\
-M_{2,1} & M_{1,1}
\end{array}\right)={ }^{t}\left(\begin{array}{l}
{\left[x_{\phi}\right]} \\
{\left[y_{\phi}\right]}
\end{array}\right) \\
& \Longleftrightarrow\left\{\begin{array}{l}
{\left[x_{\phi}\right]=M_{2,2}\left[x_{w}\right]-M_{2,1}\left[y_{w}\right]} \\
{\left[y_{\phi}\right]=-M_{1,2}\left[x_{w}\right]+M_{1,1}\left[y_{w}\right]}
\end{array} .\right.
\end{aligned}
$$

And we have $\left[x_{\phi}\right] \neq 0$ and $\left[y_{\phi}\right]=0$ in $H_{1}\left(V_{w}\right)$ by Lemma 3.8. Also we get $\left[m_{w}\right]=\left[y_{\phi}\right]$ and $\left[l_{w}\right]=\left[x_{\phi}\right]$ in $H_{1}\left(V_{w}\right)$. It completes the proof.

Proof of THEOREM 3.6. Recall that $a_{i}$ is defined by the equation $q / p=$ $\left[a_{1}, a_{2}, \cdots, a_{n}, 1\right]$. Then, we define the matrix $\mathcal{A}^{(p, q)}$ as follows:

$$
\mathcal{A}^{(p, q)}=\left\{\begin{array}{lll}
U_{L}^{a_{1}} U_{R}^{a_{2}} U_{L}^{a_{3}} \cdots U_{L}^{a_{n-2}} U_{R}^{a_{n-1}} U_{L}^{a_{n}} & (n: \text { odd }) \\
U_{L}^{a_{1}} U_{R}^{a_{2}} U_{L}^{a_{3}} \cdots U_{R}^{a_{n-2}} U_{L}^{a_{n-1}} U_{R}^{a_{n}} & (n: \text { even })
\end{array} .\right.
$$


For convenience, we denote the word $A(p, q)$ by $A$. By Theorem 3.5, we have the following relations in $H_{1}\left(\partial T_{A}\right)$ :

$$
\left[m_{A}\right]=-A_{1,2}\left[x_{A}\right]+A_{1,1}\left[y_{A}\right] ; \quad\left[l_{A}\right]=A_{2,2}\left[x_{A}\right]-A_{2,1}\left[y_{A}\right],
$$

where $A_{i, j}$ is the $(i, j)$-element of the matrix $\mathcal{A}^{(p, q)}$. Thus, we have

$$
\left[x_{A}\right]=A_{1,1}\left[l_{A}\right]+A_{2,1}\left[m_{A}\right] ; \quad\left[y_{A}\right]=A_{1,2}\left[l_{A}\right]+A_{2,2}\left[m_{A}\right] .
$$

Then, the following equation holds in $H_{1}\left(\partial T_{A}\right)$ :

$$
\begin{aligned}
{\left[z_{A}\right] } & =\left[x_{A}\right]+\left[y_{A}\right] \\
& =A_{1,1}\left[l_{A}\right]+A_{2,1}\left[m_{A}\right]+A_{1,2}\left[l_{A}\right]+A_{2,2}\left[m_{A}\right] \\
& =\left(A_{1,1}+A_{1,2}\right)\left[l_{A}\right]+\left(A_{2,1}+A_{2,2}\right)\left[m_{A}\right] .
\end{aligned}
$$

By Lemma 3.9 and the definition of $a_{i}$, we have

$$
\frac{A_{2,1}+A_{2,2}}{A_{1,1}+A_{1,2}}=\left[a_{1}, a_{2}, \cdots, a_{n}, 1\right]=\frac{q}{p} .
$$

And, two natural numbers $A_{2,1}+A_{2,2}$ and $A_{1,1}+A_{1,2}$ are coprime by Lemma 3.9. Thus, we have $A_{1,1}+A_{1,2}=p$ and $A_{2,1}+A_{2,2}=q$.

3.4. $b$-type solid torus. In this subsection, we define the b-type solid torus for an integer $b$. In $\S 5$, it will be appeared that $b$-type solid torus corresponds to an obstruction class of Seifert manifold.

For an integer $b$, the word $w(b)$ is defined as $L R^{b} \bar{L}$. Then, we consider a meridianlongitude system of the solid torus $V_{w(b)}$.

COROLLARY 3.10. For the solid torus $V_{w(b)}$, there is a meridian-longitude system $\left(m_{w(b)}, l_{w(b)}\right)$ satisfying the following conditions in $H_{1}\left(\partial V_{w(b)}\right)$ :

$$
\begin{aligned}
{\left[m_{w(b)}\right] } & =b\left[x_{w(b)}\right]+(b+1)\left[y_{w(b)}\right] ; \\
{\left[l_{w(b)}\right] } & =(-b+1)\left[x_{w(b)}\right]-b\left[y_{w(b)}\right] .
\end{aligned}
$$

Proof. We have $U_{L} U_{R}^{b} U_{\bar{L}}=\left(\begin{array}{cc}-b+1 & b \\ -b & 1+b\end{array}\right)$. Theorem 3.5 completes the proof.

THEOREM 3.11. For the solid torus $V_{w(b)}$ with the meridian-longitude system $\left(m_{w(b)}, l_{w(b)}\right)$ given by Corollary 3.10, the loop $z_{w(b)}$ is the $(1,1)$-type.

PROOF. The following relation holds in $H_{1}\left(\partial V_{w(b)}\right)$ :

$$
\begin{aligned}
{\left[z_{w(b)}\right] } & =\left[y_{w(b)}\right]+\left[x_{w(b)}\right] \\
& =\{b+(-b+1)\}\left[x_{w(b)}\right]+\{(b+1)+(-b)\}\left[y_{w(b)}\right] \\
& =b\left[x_{w(b)}\right]+(b+1)\left[y_{w(b)}\right]+(-b+1)\left[x_{w(b)}\right]+(-b)\left[y_{w(b)}\right]
\end{aligned}
$$




$$
=\left[m_{w(b)}\right]+\left[l_{w(b)}\right] .
$$

Notation 3.12. We call $V_{w(b)}$ as the b-type solid torus and denote it by $V_{1, b}$.

\section{One-vertex triangulation of Lens spaces}

In this section, we construct systematically one-vertex triangulations of lens spaces. In $\S 4.1$, we will define a homeomorphism $\varphi=\varphi\left(w, w^{\prime}\right): \partial V_{w} \rightarrow \partial V_{w^{\prime}}$ for two words $w$ and $w^{\prime}$, and consider the manifold obtained by gluing two solid tori $V_{w}$ and $V_{w^{\prime}}$ by $\varphi$, denoted by $V_{w} \cup_{\varphi} V_{w^{\prime}}$. In $\S 4.2$, we will show the following fact: for any pair of coprime natural numbers $p$ and $q$ such that $p>q$, we can choose two words $w$ and $w^{\prime}$ such that the lens space $L(p, q)$ is homeomorphic to the manifold $V_{w} \cup_{\varphi} V_{w^{\prime}}$. Then, we will consider a one-vertex triangulation of the lens space $L(p, q)$.

4.1. Gluing $\operatorname{map} \varphi$. For $i=1,2$, we denote by $U_{i}$ the boundary tori $S^{1} \times S^{1}$. Let $h_{i}$ be an embedding such that $U_{i} \backslash h_{i}(\theta) \cong \operatorname{Int}\left(D^{2}\right)$, where $\theta$ is a theta-curve shown in Figure 1 .

LEMMA 4.1. Two embedding $h_{1}$ and $h_{2}$ induces a homeomorphism $\varphi_{1,2}: U_{1} \rightarrow U_{2}$ such that $\varphi_{1,2}\left(h_{1}(X)\right)=h_{2}(X)$ for any edge $X \in\{\alpha, \beta, \gamma\}$ of $\theta$.

Proof. We define the map $\varphi^{\prime \prime}: h_{1}(\theta) \rightarrow h_{2}(\theta)$ by identifying $h_{1}(X)$ and $h_{2}(X)$, where $X=\alpha, \beta, \gamma$. Denote by $N\left(h_{i}(\theta)\right)$ a regular neighborhood of $h_{i}(\theta)$ in $U_{i}$. Then, the map $\varphi^{\prime \prime}$ can be extended to a homeomorphism $\varphi^{\prime}: N\left(h_{1}(\theta)\right) \rightarrow N\left(h_{2}(\theta)\right)$ such that $\varphi^{\prime}\left(h_{1}(X)\right)=h_{2}(X)$, where $X=\alpha, \beta, \gamma$. Since $U_{i} \backslash \varphi_{i}(\theta) \cong \operatorname{Int}\left(D^{2}\right)$, the homeomorphism $\varphi^{\prime}$ can be extended to a homeomorphism $\varphi: U_{1} \rightarrow U_{2}$ such that $\varphi\left(h_{1}(X)\right)=h_{2}(X)$, where $X=\alpha, \beta, \gamma$.

Now, we define a gluing map $\varphi$ of solid torus $V_{w}$ and $V_{w^{\prime}}$. Recall that for any solid torus $V_{w}$, a theta-curve $\theta$ (Figure 1) is embedded in $\partial V_{w}$ such that $\partial V_{w} \backslash \theta \cong \operatorname{Int}\left(D^{2}\right)$. Thus, for two words $w$ and $w^{\prime}$, a homeomorphism $\varphi\left(w, w^{\prime}\right): \partial V_{w} \rightarrow \partial V_{w^{\prime}}$ is given by Lemma 4.1. So, we define the manifold obtained by gluing $V_{w}$ and $V_{w^{\prime}}$ by $\varphi\left(w, w^{\prime}\right)$, denoted by $V_{w} \cup_{\varphi} V_{w^{\prime}}$. In the following subsection, we consider the manifold $V_{w} \cup_{\varphi} V_{w^{\prime}}$.

4.2. The manifold obtained by gluing $0 V_{w}$ and $V_{w^{\prime}}$ by $\varphi$. Let $p, q$ be a pair of coprime natural numbers such that $p>q$. There are many pairs of two words $\left(w, w^{\prime}\right)$ such that $V_{w} \cup_{\varphi} V_{w^{\prime}} \cong L(p, q)$, where $L(p, q)$ is a lens space. Then, the following theorem gives us a pair $\left(w, w^{\prime}\right)$, say $w=\bar{L}$ and $w^{\prime}=A(p, q)$, where the word $A(p, q)$ is defined in Theorem 3.6.

THEOREM 4.2. The manifold $V_{\bar{L}} \cup_{\varphi} V_{A(p, q)}$ is homeomorphic to the lens space $L(p, q)$. In particular, $V_{\bar{L}} \cup_{\varphi} V_{\phi} \cong S^{3}$ and $V_{\bar{L}} \cup_{\varphi} V_{\bar{L}} \cong S^{2} \times S^{1}$. 
Proof. For convenience, we denote the word $A(p, q)$ by $A$. By Theorem 3.5, there are meridian-longitude systems $\left(m_{\bar{L}}, l_{\bar{L}}\right)$ and $\left(m_{A}, l_{A}\right)$ of the solid tori $V_{\bar{L}}$ and $V_{A}$ satisfying the following relations in $H_{1}\left(\partial V_{L}\right)$ and $H_{1}\left(\partial V_{A}\right)$ respectively:

$$
\begin{aligned}
{\left[m_{\bar{L}}\right] } & =-\left[x_{\bar{L}}\right]+\left[y_{\bar{L}}\right] ; \\
{\left[m_{A}\right] } & =-A_{1,2}\left[x_{A}\right]+A_{1,1}\left[y_{A}\right] ; \\
{\left[l_{A}\right] } & =A_{2,2}\left[x_{A}\right]-A_{2,1}\left[y_{A}\right],
\end{aligned}
$$

where $A_{i, j}$ is the $(i, j)$-element of the matrix $\mathcal{A}^{(p, q)}$, where $\mathcal{A}^{(p, q)}$ is defined in the proof of Theorem 3.6. According to the definition of the gluing map $\varphi: \partial V_{\bar{L}} \rightarrow \partial V_{A}$, we obtain

$$
\varphi\left(x_{\bar{L}}\right)=x_{A} ; \quad \varphi\left(y_{\bar{L}}\right)=y_{A} .
$$

Let $\varphi^{\#}: H_{1}\left(\partial V_{\bar{L}}\right) \rightarrow H_{1}\left(\partial V_{A}\right)$ be the isomorphism induced from the homeomorphism $\varphi$. Using the equations (1), (2), (3) and (4), we have the following relation in $H_{1}\left(\partial V_{A}\right)$.

$$
\begin{aligned}
{\left[\varphi\left(m_{\bar{L}}\right)\right] } & =\varphi^{\#}\left(\left[m_{\bar{L}}\right]\right)=\varphi^{\#}\left(\left[y_{\bar{L}}\right]\right)+\varphi^{\#}\left(\left[x_{\bar{L}}\right]\right)=\left[\varphi\left(y_{\bar{L}}\right)\right]+\left[\varphi\left(x_{\bar{L}}\right)\right] \\
& =\left[y_{A}\right]+\left[x_{A}\right]=A_{1,1}\left[l_{A}\right]+A_{2,1}\left[m_{A}\right]+\left(A_{1,2}\left[l_{A}\right]+A_{2,2}\left[m_{A}\right]\right) \\
& =\left(A_{1,1}+A_{1,2}\right)\left[l_{A}\right]+\left(A_{2,1}+A_{2,2}\right)\left[m_{A}\right] .
\end{aligned}
$$

By Lemma 3.9, two natural numbers $A_{1,1}+A_{1,2}$ and $A_{2,1}+A_{2,2}$ are coprime and

$$
\frac{A_{2,1}+A_{2,2}}{A_{1,1}+A_{1,2}}=\left[a_{1}, a_{2}, \cdots, a_{n}, 1\right]=\frac{q}{p} .
$$

Thereby, the manifold $V_{\bar{L}} \cup_{\varphi} V_{A}$ is homeomorphic to the lens space $L(p, q)$.

At last, we will show $V_{\bar{L}} \cup_{\varphi} V_{\phi} \cong S^{3}$ and $V_{\bar{L}} \cup_{\varphi} V_{\bar{L}} \cong S^{2} \times S^{1}$. By theorem 3.5, we get $\left[\varphi\left(m_{\bar{L}}\right)\right]=\left[m_{\phi}\right]+\left[l_{\phi}\right]$ and $\left[\varphi\left(m_{\bar{L}}\right)\right]=\left[m_{\bar{L}}\right]$. It completes the proof.

Recall the definition $D_{w}$, see $\S 2.1$ and 2.2. Let $P$ be the 2-manifold obtained by gluing $\left(D_{\bar{L}} / f_{\bar{L}}\right)$ and $\left(D_{A(p, q)} / f_{A(p, q)}\right)$ with $\varphi$. Then, $P$ is a special spine of the lens space $L(p, q) \cong$ $V_{\bar{L}} \cup_{\varphi} V_{A(p, q)}$, Thus, the dual complex of $P$ in $V_{\bar{L}} \cup_{\varphi} V_{A(p, q)}$ is a one-vertex triangulation of $L(p, q)$.

\section{One-vertex triangulation of Seifert manifolds}

In this section, we construct systematically one-vertex triangulations of all orientable Seifert manifolds with orientable base. Our construction is based on the following fact: any orientable Seifert manifold is obtained by gluing the compact manifolds $M_{n}, J$ and $V_{p, q}$ which are homeomorphic to $\left(S^{2}-\coprod_{i=1}^{n} \operatorname{Int}\left(D_{i}^{2}\right)\right) \times S^{1},\left(S^{1} \times S^{1}-\operatorname{Int}\left(D^{2}\right)\right) \times S^{1}$ and $(p, q)$ type fibered solid torus.

In $\S 5.2$ and 5.3, we consider the manifolds $J$ and $M_{n}$ respectively. In $\S 5.4$, we define the manifold $\mathcal{M}$ obtained by gluing $V_{p_{i}, q_{i}}, V_{b}, J$ and $M_{n}$, see Notation 3.7 and 3.12 about $V_{p_{i}, q_{i}}$ and $V_{b}$, and consider a fiber structure and a one-vertex triangulation of $\mathcal{M}$. 


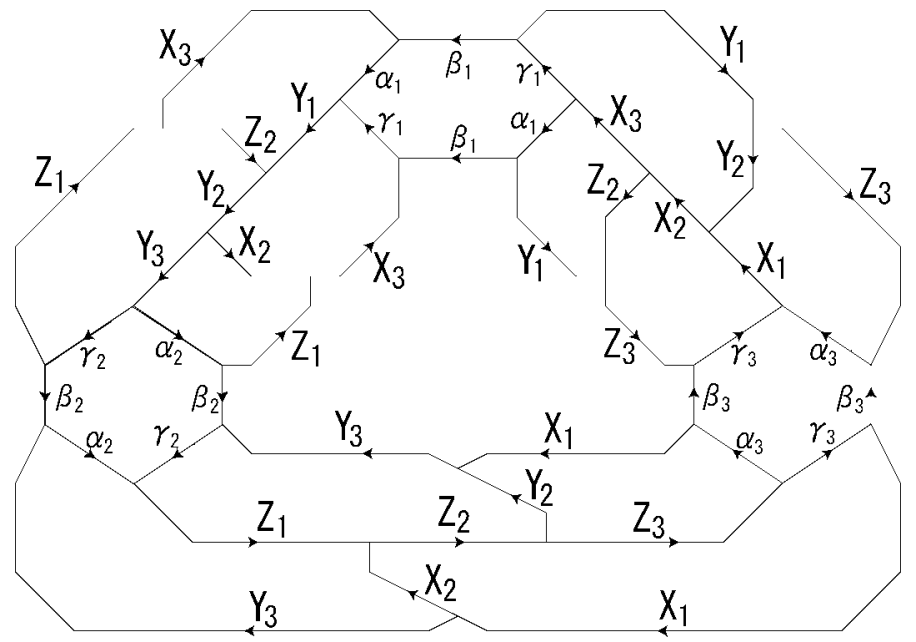

FIGURE 17. $\mathcal{K}$.

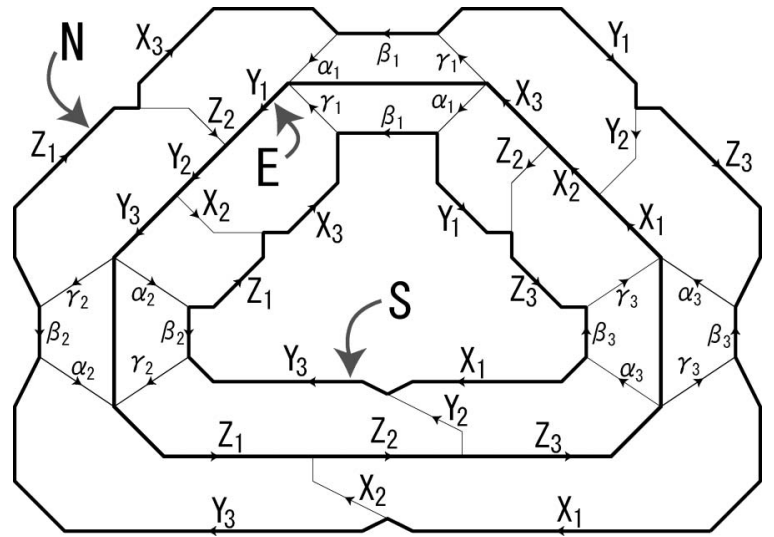

FIGURE 18. $\quad E:=\partial B^{3} \cap\{z=0\}, S:=\partial B^{3} \cap\left\{z=\frac{1}{\sqrt{2}}\right\}, N:=\partial B^{3} \cap\left\{z=-\frac{1}{\sqrt{2}}\right\}$.

5.1. $\quad\left(S^{2}-\coprod_{i=1}^{3} \operatorname{Int}\left(D_{i}^{2}\right)\right) \times S^{1}$. We use the notation $\mathcal{K}$ for the labeled 3-regular graph shown in Figure 17. Suppose that $\mathcal{K}$ is embedded in $\partial B^{3}$. We denote by $D_{i}$ the 2-disc bounded by the circle $\alpha_{i} \beta_{i} \gamma_{i} \overline{\alpha_{i}} \overline{\beta_{i}} \overline{\gamma_{i}}$ in $S^{2}\left(=\partial B^{3}\right)$. Then, by removing $D_{1}, D_{2}$ and $D_{3}$ from $S^{2}\left(=\partial B^{3}\right)$, we get a 2 -sphere with three holes. We denote it by $D_{\mathcal{K}}$. Analyzing the notion of an identification map for a word diagram in $\S 2$, we can consider an identification map $f_{\mathcal{K}}$ on $\partial B^{3}$ and a compact 3-manifold $K=B^{3} / f_{\mathcal{K}}$.

Proposition 5.1. The manifold $K$ is homeomorphic to $\left(S^{2}-\bigsqcup_{i=1}^{3} \operatorname{Int}\left(D_{i}^{2}\right)\right) \times S^{1}$. 
PROOF. Without loss of generality, we assume the following three conditions (a),(b),(c) about the embedding of the graph $\mathcal{K}$ in $S^{2}\left(=\partial B^{3}\right)$ :

(a) $B^{3}=\left\{(x, y, z) \in \mathbf{R}^{3} \mid x^{2}+y^{2}+z^{2} \leq 1\right\}$;

(b) $\partial B^{3} \cap\{z=0\}, \partial B^{3} \cap\left\{z=\frac{1}{\sqrt{2}}\right\}$ and $\partial B^{3} \cap\left\{z=-\frac{1}{\sqrt{2}}\right\}$ are the bold circles $E, N$ and $S$ shown in Figure 18 respectively.

We denote by $A_{N}(a, b), A_{S}(a, b)$ the arcs in $\partial B^{3}$ defined by

$$
\begin{aligned}
& A_{+}(a, b):=\left\{(x, y, z) \mid x=\frac{1}{\sqrt{2}} \cos \theta, y=\frac{1}{\sqrt{2}} \sin \theta, z=\frac{1}{\sqrt{2}}, a<\theta<b\right\} \\
& A_{-}(a, b):=\left\{(x, y, z) \mid x=\frac{1}{\sqrt{2}} \cos \theta, y=\frac{1}{\sqrt{2}} \sin \theta, z=-\frac{1}{\sqrt{2}}, a<\theta<b\right\} .
\end{aligned}
$$

(c) The edges in the circles $S$ and $N$ satisfy the following conditions:

$$
\begin{array}{rlrl}
Y_{1} & =A_{ \pm}\left(0, \frac{2}{9} \pi\right) ; & Z_{3}=A_{ \pm}\left(\frac{2}{9} \pi, \frac{4}{9} \pi\right) ; & \beta_{3}=A_{ \pm}\left(\frac{4}{9} \pi, \frac{6}{9} \pi\right) ; \\
X_{1}=A_{ \pm}\left(\frac{6}{9} \pi, \frac{8}{9} \pi\right) ; & Y_{3}=A_{ \pm}\left(\frac{8}{9} \pi, \frac{10}{9} \pi\right) ; & \beta_{2}=A_{ \pm}\left(\frac{10}{9} \pi, \frac{12}{9} \pi\right) ; \\
Z_{1}=A_{ \pm}\left(\frac{12}{9} \pi, \frac{14}{9} \pi\right) ; & X_{3}=A_{ \pm}\left(\frac{14}{9} \pi, \frac{16}{9} \pi\right) ; & \beta_{1}=E_{ \pm}\left(\frac{16}{9} \pi, 2 \pi\right) .
\end{array}
$$

Consider the flow generated by the vector field $\partial / \partial z$ on $B^{3}$. A point $a_{0}$ in Figure 19 is mapped by the identification map $f_{\mathcal{K}}$ to $a_{1}$. And it is moved by the flow $\partial / \partial z$ and arrives at $a_{2}$. After that, it is mapped to $a_{3}$ by $f_{\mathcal{K}}$ and it turns back to the same point $a$ by the flow $\partial / \partial z$. Also, any point in $\operatorname{Int}\left(A_{1}\right)$ turns back to the same point. Similarly, any point in $\operatorname{Int}\left(B_{1}\right), \operatorname{Int}\left(C_{1}\right)$, $\operatorname{Int}\left(D_{1}\right)$ and $\operatorname{Int}\left(E_{1}\right)$ turns back to the same point respectively.

Then, by the method shown in $\S 4$ in [6], we know that $D_{\mathcal{K}}^{\prime} / f_{\mathcal{K}}$ is the orbit space obtained by $f_{\mathcal{K}}$ and $\partial / \partial z$, see Figure 20, and it is embedded in $K$. Since $D_{\mathcal{K}}^{\prime} / f_{\mathcal{K}}$ is homeomorphic to $S^{2}-\bigsqcup_{i=1}^{3} \operatorname{Int}\left(D_{i}^{2}\right)$, we get the manifold $\left(S^{2}-\bigsqcup_{i=1}^{3} \operatorname{Int}\left(D_{i}^{2}\right)\right) \times I$ by cutting $K$ along $D_{\mathcal{K}}^{\prime} / f_{\mathcal{K}}$, Thus, the manifold $K$ is homeomorphic to $\left(S^{2}-\coprod_{i=1}^{3} \operatorname{Int}\left(D_{i}^{2}\right)\right) \times S^{1}$.

We define a fiber structure of $K$ by the vector field $\partial / \partial z$. Thus, the loop $\{(x, y, z) \mid$ $x=y=0,-1 \leq z \leq 1\} / f_{\mathcal{K}}$ is a fiber of $K$. So, the loops $\gamma_{k} \overline{\alpha_{k}}$ are homotopic to fibers of $K$, where $k=1,2,3$.

5.2. $\left(S^{1} \times S^{1}-\operatorname{Int}\left(D^{2}\right)\right) \times S^{1}$. We consider the manifold $\left(S^{1} \times S^{1}-\operatorname{Int}\left(D^{2}\right)\right) \times S^{1}$. We use the notation $D_{\mathcal{J}}$ for the disc shown in Figure 21. Assume that $D_{\mathcal{J}}$ is embedded in $S^{2}\left(=\partial B^{3}\right)$. Then, an identification map $f_{\mathcal{J}}$ on $S^{2}\left(=\partial B^{3}\right)$ is induced by identifying the directed labeled edges of the $D_{\mathcal{J}}$ similar to $\S 5.1$. Then, we consider a compact 3-manifold $J=B^{3} / f_{\mathcal{J}}$.

Proposition 5.2. The manifold $J$ is homeomorphic to $\left(S^{1} \times S^{1}-\operatorname{Int}\left(D^{2}\right)\right) \times S^{1}$. 


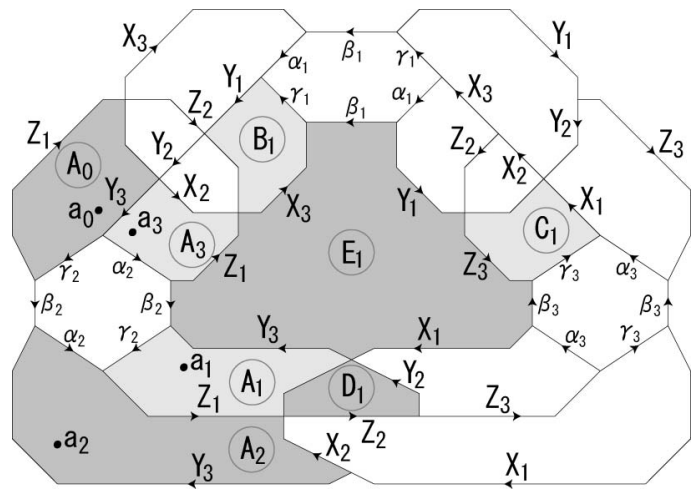

FIGURE 19. A, B, C, D and E.

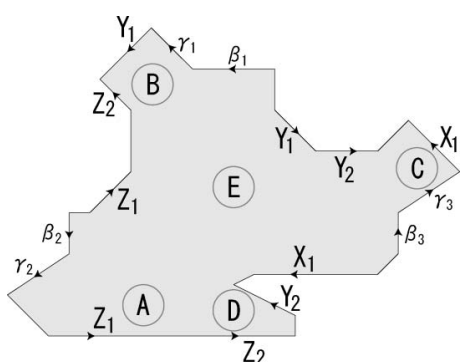

FIGURE 20. $D_{\mathcal{K}}^{\prime}$.

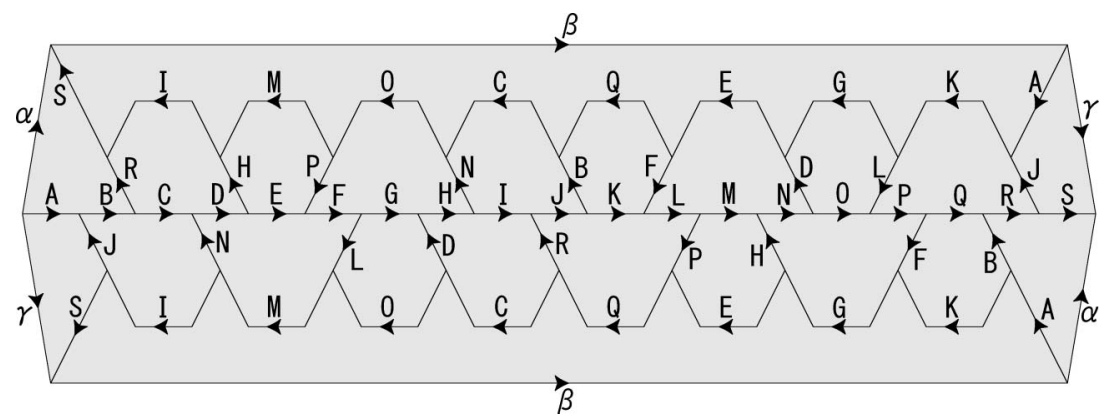

FIGURE 21. $D_{\mathcal{J}}$.

Proof. The proof is similar to Proposition 5.1. Suppose that the $D_{\mathcal{J}}$ is embedded in the boundary of the unit ball. We consider the orbit space generated by the flow $\partial / \partial z$. Then, we have $D_{\mathcal{J}}^{\prime} / f_{\mathcal{J}}$ is homeomorphic to $S^{1} \times S^{1}-\operatorname{Int}\left(D^{2}\right)$. Thus, $J$ is homeomorphic to $\left(S^{1} \times S^{1}-\operatorname{Int}\left(D^{2}\right)\right) \times S^{1}$.

5.3. $\quad\left(S^{2}-\coprod_{i=1}^{n} \operatorname{Int}\left(D_{i}^{2}\right)\right) \times S^{1}$. For a natural number $n \geq 3$, we define the manifold $M_{n}$. We prepare $(n-2)$ - copies $K^{(3)}, K^{(4)}, \cdots, K^{(n)}$ of $K$, where $K$ is homeomorphic to $\left(S^{2}-\coprod_{i=1}^{3} \operatorname{Int}\left(D_{i}^{2}\right)\right) \times S^{1}$ defined in $\S 5$.1. For $j=3,4, \cdots, n,\left(C_{1}^{(j)}, \theta_{1}\right),\left(C_{2}^{(j)}, \theta_{2}\right)$ and $\left(C_{3}^{(j)}, \theta_{3}\right)$ are pairs of a boundary component $C_{i}^{(j)}$ of $K^{(j)}$ and the theta-curve $\theta_{i}$ (Figure 22) embedded in $C_{i}^{(j)}$, where $C_{i}^{(j)}$ is homeomorphic to $S^{1} \times S^{1}$. Since the theta-curve $\theta_{i}$ is embedded in $C_{i}^{(j)}$ such that $C_{i}^{(j)} \backslash \theta_{i} \cong \operatorname{Int}\left(D^{2}\right)$, we can define homeomorphisms $\varphi^{(j)}$ : $C_{3}^{(j)} \rightarrow C_{1}^{(j+1)}$ by Lemma 4.1 for $j=3,4, \cdots, n-1$. By using $\varphi^{(j)}, M_{n}$ is defined as $K^{(3)} \cup_{\varphi^{(3)}} K^{(4)} \cup_{\varphi^{(4)}} \cdots \cup_{\varphi^{(n-1)}} K^{(n)}$. 


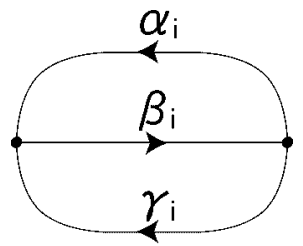

FIGURE 22. $\theta_{i}$.

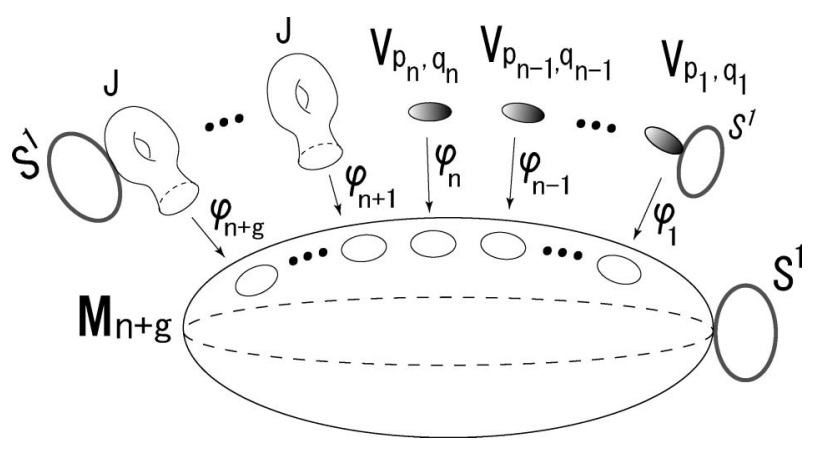

FIGURE 23. Gluing maps $\left\{\varphi_{i}\right\}$.

Note that the homeomorphisms $\varphi^{(j)}$ satisfy the conditions $\varphi^{(j)}\left(\alpha_{3}\right)=\alpha_{1}, \varphi^{(j)}\left(\beta_{3}\right)=\beta_{1}$ and $\varphi^{(j)}\left(\gamma_{3}\right)=\gamma_{1}$, where $\theta_{i}=\alpha_{i} \cup \beta_{i} \cup \gamma_{i}$. Thus, the homeomorphism $\varphi^{(j)}$ is a fiber preserving, and the manifold $M_{n}$ is homeomorphic to $\left(S^{2}-\coprod_{i=1}^{n} \operatorname{Int}\left(D_{i}^{2}\right)\right) \times S^{1}$.

5.4. The manifold obtained by gluing $J, V_{b}, V_{p, q}$ and $M_{n}$. Let $g$ and $n$ be integers such that $g \geq 0$ and $n \geq 3$. Then, we consider the manifold $M_{n+g}$. As shown in $\S 5.3$, a thetacurve $\theta_{i}$ (Figure 22) is embedded in each boundary component $C_{i}$ of $M_{n+g}$ such that $C_{i} \backslash \theta_{i} \cong$ Int $\left(D^{2}\right)$, where $i=1,2, \cdots, n+g$. Recall the manifold $J, V_{b}$ and $V_{p, q}$ defined in $\S 5.2, \S 3.4$ and $\S 3.3$ respectively. The boundary of each manifolds $J, V_{b}$ and $V_{p, q}$ is homeomorphic to $S^{1} \times S^{1}$ and the theta-curve $\theta$ (Figure 1) is embedded in such that $\partial X \backslash \theta \cong \operatorname{Int}\left(D_{i}^{2}\right)$, where $X=J, V_{b}, V_{p, q}$. Thus, by Lemma 4.1 there are $n+g$ homeomorphisms $\left\{\varphi_{i}\right\}_{i=1}^{n+g}$

$$
\varphi_{i}:= \begin{cases}\partial V_{p_{i}, q_{i}} \rightarrow C_{i} & (1 \leq i \leq n), \\ \partial J \rightarrow C_{i} & (n+1 \leq i \leq n+g),\end{cases}
$$

see Figure 23. Thus, we define the manifold obtained by gluing $\coprod_{i=1}^{n} V_{p_{i}, q_{i}}, \coprod_{i=n+1}^{n+g} J$ and $M_{n+g}$, denoted by $M\left(F_{g},\left(p_{1}, q_{1}\right), \cdots,\left(p_{n}, q_{n}\right)\right)$. Then, we consider fiber structure of it.

1. In the case $1 \leq i \leq n$

(a) $p_{i} \neq 1$ 
Recall $V_{p_{i}, q_{i}}$, see Notation 3.7. By the definition of the gluing map $\varphi_{i}$, we get $\varphi_{i}^{-1}\left(\gamma_{i} \overline{\alpha_{i}}\right)=\gamma \bar{\alpha}$. Since the loop $\gamma_{i} \overline{\alpha_{i}}$ is a fiber of the manifold $M_{n+g}$, we regard the loop $\gamma \bar{\alpha}$ in $\partial V_{p_{i}, q_{i}}$ as a fiber of the solid torus $V_{p_{i}, q_{i}}$. That is, we decide a fiber structure of $V_{p_{i}, q_{i}}$ by the loop $\gamma \bar{\alpha}$. By Theorem 3.6, we have $[\gamma \bar{\alpha}]=p_{i}\left[l_{i}\right]+q_{i}\left[m_{i}\right]$ in $H_{1}\left(\partial V_{p_{i}, q_{i}}\right)$. So, $V_{p_{i}, q_{i}}$ is $\left(p_{i}, q_{i}\right)$-type fibered solid torus. Thus, the core of the solid torus $V_{p_{i}, q_{i}}$ is the $\left(p_{i}, q_{i}\right)$-type singular fiber.

(b) $p_{i}=1$

Recall $V_{1, b}$, see Notation 3.12. We get the following equation in $H_{1}\left(C_{i}\right)$ by Corollary 3.10, where $C_{i}$ is a boundary component of the manifold $K_{n+g}$.

$$
\begin{aligned}
{\left[\varphi_{i}\left(m_{w(b)}\right)\right] } & =\varphi_{i}^{\#}\left(\left[m_{w(b)}\right]\right) \\
& =\varphi_{i}^{\#}\left(b\left[x_{w(b)}\right]+(b+1)\left[y_{w(b)}\right]\right) \\
& =b \varphi^{\#}\left(\left[x_{w(b)}\right]\right)+(b+1) \varphi^{\#}\left(\left[y_{w(b)}\right]\right) \\
& =b \varphi^{\#}([\overline{\alpha \beta}])+(b+1) \varphi^{\#}([\gamma \beta]) \\
& =b \varphi^{\#}([\bar{\beta} \bar{\gamma} \gamma \bar{\alpha}])+(b+1) \varphi^{\#}([\gamma \beta]) \\
& =\varphi^{\#}([\gamma \beta])+b \varphi^{\#}([\gamma \bar{\alpha}]) \\
& =\left[\gamma_{i} \beta_{i}\right]+b\left[\gamma_{i} \overline{\alpha_{i}}\right] .
\end{aligned}
$$

Recall the following fact shown in the proof of Proposition 5.1: the loop $\gamma_{i} \beta_{i}$ is the intersection of $C_{i}$ and a cross section of $M_{n+g}$. Since the loop $\gamma_{i} \bar{\alpha}_{i}$ is a fiber of $M_{n+g}$, the core of $V_{b}$ is a regular fiber corresponding to the obstruction class $b$.

2. In the case $n+1 \leq i \leq n+g$

The intersection of cross sections of $J$ and $M_{n+g}$ and $\partial J$ and $\partial M_{n+g}$ are the loops $\beta \gamma$ and $\beta_{i} \gamma_{i}$ respectively. Since the conditions $\varphi_{i}(\gamma \beta)=\gamma_{i} \beta_{i}$ holds, the genus of base space of $M\left(F_{g},\left(p_{1}, q_{1}\right), \cdots,\left(p_{n}, q_{n}\right)\right)$ is equal to $g$.

A one-vertex triangulation of $M:=M\left(F_{g},\left(p_{1}, q_{1}\right), \cdots,\left(p_{n}, q_{n}\right)\right)$ is constructed similar to the lens space in $\S 4.2$. That is, a one-vertex triangulation of $M$ is the dual complex of the 2-manifold obtained by gluing $\bigsqcup_{i=1}^{n} D_{w\left(p_{i}, q_{i}\right)} / f_{w\left(p_{i}, q_{i}\right)}, \coprod_{i=1}^{g} D_{\mathcal{J}} / f_{\mathcal{J}}$ and $\coprod_{i=3}^{n+g} D_{\mathcal{K}}^{(i)} / f_{\mathcal{K}}$.

EXAMPLE.

1. Quaternionic space

$$
S\left(S^{2},-1 ;(2,1),(2,1),(2,1)\right) \cong M\left(F_{0},(2,1),(2,1),(2,1)\right)
$$

2. Brieskorn manifold $\Sigma(2,3,5)$

$$
S\left(S^{2},-1 ;(2,1),(3,1),(5,1)\right) \cong M\left(F_{0},(2,1),(3,1),(5,1)\right)
$$

3. $S\left(S^{2},-2 ;(2,1),(5,3),(7,5)\right) \cong M\left(F_{0},(2,1),(5,2),(7,3)\right)$ 


\section{References}

[ 1 ] V. G. TURAEV and O. Y. VIRo, State sum invariant of 3-manifolds and quantum $6 j$-symbols, Topology, 31 (1992), 865-902.

[ 2 ] Peter ORLIK, Seifert manifolds, Lecture Notes in Mathematics 291, Springer-Verlag.

[ 3 ] T. TANIGUCHI, Turaev-Viro invariant of Seifert manifolds, preprint.

[4 ] M. ENDO and I. IsHII, A new complexity for 3-manifolds, to appear in Japanese J. Math. preprint.

[5] Sergei Matveev, Algorithmic Topology and Classification of 3-manifolds, Springer.

[ 6 ] I. IsHII, Flow-spine and Seifert fibred structure of 3-manifolds, Tokyo J. Math., 11 (1988), 95-104.

[ 7 ] H. IKEDA and Y. INOUE, Invitation to DS-diagrams, Kobe J Math., 2 (1985), 169-186.

[ 8 ] K. YокоYама, On DS-diagrams of lens spaces, Topology and computer science, Edited by S.Suzuki, Kinokuniya Company Ltd., (1987), 171-192.

Present Addresses:

TAIJI TANIGUCHI

Department of Mathematics, Faculty of Science and TeChnology, KeIo University, KOHOKU-KU, YOKOHAMA, 223-8522 JAPAN.

e-mal: taiji@math.keio.ac.jp

Keiko TSUboi

Department of Mathematics, Dokkyo Saitama High School / Junior High School, ONMASHINDEn, Koshigaya, SAITAMa, 343-0037 JAPAN.

e-mal: tsubokei@hotmail.com

MASAKATSU YAMASHITA

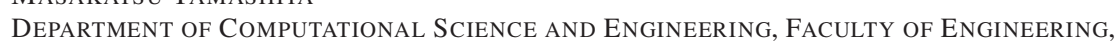
TOYO UNIVERSITY,

KAWAGOE-SHI, SAITAMA, 350-8585 JAPAN.

e-mal: yamasita@eng.toyo.ac.jp 